\title{
Heterogeneous nucleation of a droplet pinned at a chemically inhomogeneous substrate: A simulation study of the two-dimensional Ising case
}

\author{
Marta L. Trobo, ${ }^{1,2}$ Ezequiel V. Albano, ${ }^{1,3}$ and Kurt Binder ${ }^{4}$ \\ ${ }^{1}$ Instituto de Física de Líquidos y Sistemas Biológicos (IFLYSIB), CCT-CONICET La Plata, \\ UNLP, Calle 59 Nro. 789, 1900 La Plata, Argentina \\ ${ }^{2}$ Departamento de Ciencias Básicas, Facultad de Ingeniería, Universidad Nacional de La Plata, \\ La Plata, Argentina \\ ${ }^{3}$ Departamento de Física, Facultad de Ciencias Exactas, Universidad Nacional de La Plata, La Plata, Argentina \\ ${ }^{4}$ Institut für Physik, Johannes Gutenberg-Universität Mainz, Staudinger Weg 7, D-55099 Mainz, Germany
}

(Received 21 November 2017; accepted 12 February 2018; published online 15 March 2018)

\begin{abstract}
Heterogeneous nucleation is studied by Monte Carlo simulations and phenomenological theory, using the two-dimensional lattice gas model with suitable boundary fields. A chemical inhomogeneity of length $b$ at one boundary favors the liquid phase, while elsewhere the vapor is favored. Switching on the bulk field $H_{b}$ favoring the liquid, nucleation and growth of the liquid phase starting from the region of the chemical inhomogeneity are analyzed. Three regimes occur: for small fields, $H_{b}<H_{b}^{c r i t}$, the critical droplet radius is so large that a critical droplet having the contact angle $\theta_{c}$ required by Young's equation in the region of the chemical inhomogeneity does not yet "fit" there since the baseline length of the circle-cut sphere droplet would exceed $b$. For $H_{b}^{\text {crit }}<H_{b}<H_{b}^{*}$, such droplets fit inside the inhomogeneity and are indeed found in simulations with large enough observation times, but these droplets remain pinned to the chemical inhomogeneity when their baseline has grown to the length $b$. Assuming that these pinned droplets have a circle cut shape and effective contact angles $\theta_{\text {eff }}$ in the regime $\theta_{c}<\theta_{\text {eff }}<\pi / 2$, the density excess due to these droplets can be predicted and is found to be in reasonable agreement with the simulation results. On general grounds, one can predict that the effective contact angle $\theta_{\text {eff }}$ and the excess density of the droplets, scaled by $b$, are functions of the product $b H_{b}$ but do not depend on both variables separately. Since the free energy barrier for the "depinning" of the droplet (i.e., growth of $\theta_{\text {eff }}$ to $\pi-\theta_{c}$ ) vanishes when $\theta_{\text {eff }}$ approaches $\pi / 2$, in practice only angles $\theta_{\text {eff }}$ up to about $\theta_{\text {eff }}^{\max } \simeq 70^{\circ}$ were observed. For larger fields $\left(H_{b}>H_{b}^{*}\right)$, the droplets nucleated at the chemical inhomogeneity grow to the full system size. While the relaxation time for the growth scales as $\tau_{G} \propto H_{b}^{-1}$, the nucleation time $\tau_{N}$ scales as $\ln \tau_{N} \propto H_{b}^{-1}$. However, the prefactor in the latter relation, as evaluated for our simulations results, is not in accord with an extension of the Volmer-Turnbull theory to two-dimensions, when the theoretical contact angle $\theta_{c}$ is used. Published by AIP Publishing. https://doi.org/10.1063/1.5016612
\end{abstract}

\section{INTRODUCTION}

When thermodynamic variables such as temperature $T$, pressure $p$, or external fields (e.g., a magnetic field $H$ ) are varied, discontinuous changes in the state of matter can occur. Examples of such so-called first-order phase transitions ${ }^{1,2}$ range from the condensation of water, melting of ice, crystal formation in solidifying melts, etc., to the magnetization reversal of ferromagnetic devices. These phenomena are of great importance not only for condensed matter physics but also for the atmospheric sciences, geosciences, and materials science as well as for numerous technical applications. However, a common feature of all these phase changes is that they are triggered by nucleation events; i.e., on the background of the old (metastable) phase, a nanoscopically small nucleus of the new phase needs to be formed, and such nucleation phenomena are rare events since a high free energy barrier needs to be crossed. ${ }^{2-6}$ Actually, for most conditions of practical interest, the spontaneous formation of nuclei by statistical fluctuations, i.e., the so-called "homogeneous nucleation," involves too high barriers and cannot happen. By contrast, "heterogeneous nucleation" at defects, e.g., condensation of water droplets on dust particles in the atmosphere, or surface-induced crystallization starting at the walls of a container, etc., occurs much more frequently. Also, processes such as the formation of dew droplets on car windows or plants are familiar from everyday life. ${ }^{7}$ However, the nanoscopic size of the nucleus, which typically contains only a few hundred atoms or molecules, is a stumbling block already for the theoretical description of homogeneous nucleation. ${ }^{2-6}$ The large variety of defects that can cause heterogeneous nucleation makes a comprehensive description even more difficult; see, e.g., Ref. 8. Thus theoretical work on heterogeneous nucleation is rather scarce, ${ }^{9-32}$ while theoretical work on homogeneous nucleation is abundant; see, e.g., Ref. 33 for an overview of work done for Ising/lattice gas models.

In this paper, we reconsider the problem of heterogeneous nucleation on flat substrates by focusing on a chemically 
inhomogeneous surface, where nucleation preferentially occurs in a region of finite (nanoscopic) linear dimension $b$, cf. Fig. 1. There are several motivations for such a choice: (i) chemically structured surfaces are useful for many applications in nanotechnology such as the fabrication of nanodevices, for the processing of nanoscopic amounts of matter ("lab in a chip"), etc. ${ }^{34,35}$ (ii) Since $b$ is comparable to the size of the nucleus formed in a single heterogeneous nucleation event, it is straightforward to study the characteristics of such isolated nucleation events by computer simulation in great detail. By contrast, when one studies nucleation in a system with a homogeneous macroscopic surface, surface-attached nuclei can occur anywhere on this substrate and one easily reaches conditions where several nuclei form and compete during their growth. This case is familiar from studies of homogeneous nucleation (e.g., Refs. 3, 33, and 36-38), and observing the lifetime of metastable states in this limit of multi-nuclei formation and growth allows only rather indirect conclusions on the nucleation rates and nucleation barriers. (iii) Due to the fact that dust or soot particles at which nucleation happens in the atmosphere are often of $\mu \mathrm{m}$ size only and have irregular shapes and need not be chemically homogeneous, it is of practical interest to study cases where conditions favorable for heterogeneous nucleation are limited to regions of nanoscopic extent. Of course, also other types of localized defects are suitable to study isolated heterogeneous nucleation events; e.g., in square lattices with free boundary conditions, nucleation starts at the corners of the square. ${ }^{39}$

Of course, for this problem there occurs a challenging interplay between surface effects due to the substrate and interfacial effects of the material forming the nucleus; also,

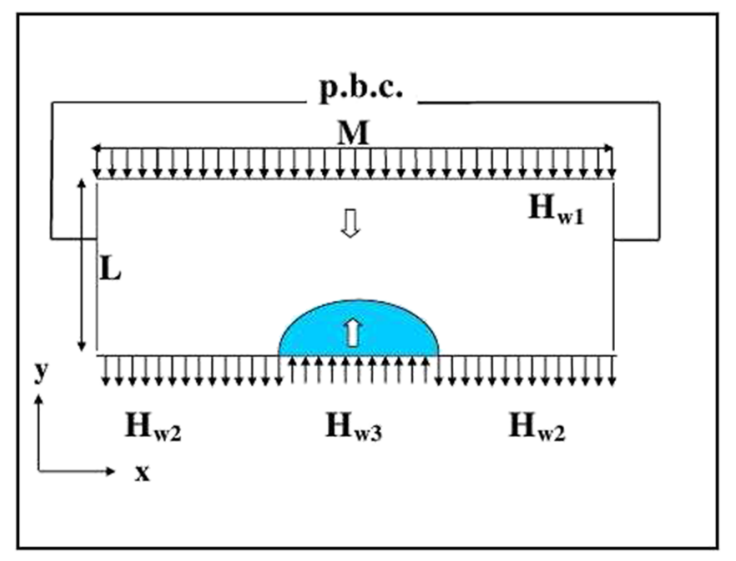

FIG. 1. Schematic description of the system geometry. We choose a rectangular $M \times L$ lattice with periodic boundary conditions in the $x$-direction and surface fields acting on the first and last row of spins in the $y$-direction. The sign of the surface fields is indicated by the arrows. The surface field on the top row $\left(H_{w 1}\right)$ and outside the inhomogeneity at the bottom row $\left(H_{w 2}\right)$ are both chosen negative so that a negative magnetization in the bulk of the system is stable (thus this area is left in white and the wide arrow indicates the negative magnetization). The strength $H_{w 1}$ of the surface field on top is chosen smaller $\left(\left|H_{w 1}\right|=\left|H_{w 2}\right| / 4\right)$, but for the large linear dimensions chosen the precise choice of $H_{w 1}$ does not matter with respect to the properties of the droplet. Also, for $b$ sites at the bottom row, a positive field $H_{w 3}=\left|H_{w 2}\right|$ is chosen, and a positive bulk field is applied throughout the sample that favors the heterogeneous nucleation of the droplets within the wall inhomogeneity (the droplet area is colored and surrounds a wide arrow pointing up showing the prevalent positive magnetization in the pinned droplet). statistical fluctuations and finite-size effects play a role. Therefore, the development of an analytical theory for the treatment of such problems is very difficult. ${ }^{40-42}$ In the present work, we hence restrict our attention to an approach by Monte Carlo simulation ${ }^{43}$ of a simple model, namely, the Ising/lattice gas model. As has been discussed elsewhere, ${ }^{33}$ even this simple model presents severe difficulties due to the incomplete knowledge of the anisotropy of the interfacial tension between bulk coexisting phases. Also understanding of wetting phenomena, e.g., contact angles of macroscopic sessile droplets at walls, ${ }^{44}$ is a problem in the three-dimensional case (see, e.g., Refs. 45-47). Thus, we focus here on the lattice gas/Ising model in $d=2$ dimensions, for which both bulk and interfacial phenomena including wetting behavior $^{48-54}$ are well understood. Recently, we have already studied droplets pinned at chemically heterogeneous substrates under conditions of bulk phase coexistence. ${ }^{55}$ This knowledge also is useful for the present work where we consider such droplets under out-of-equilibrium conditions and the dynamics of the resulting growth process during nucleation events.

The outline of our paper is as follows: In Sec. II, we precisely characterize the model and the simulation method. In Sec. III, we present our results for wall-attached precursor droplets which are in metastable equilibrium during the "observation time" of the simulation. Furthermore, we discuss a scaling description in terms of the variables $b$ (spatial extent of the inhomogeneity) and bulk magnetic field $H_{b}$ (which characterizes the "distance" of the metastable state from phase coexistence that occurs in the bulk at $H_{b}=0$, of course). In Sec. IV, we present a phenomenological theory of pinned metastable droplets which have a baseline $b$ and a non-equilibrium contact angle controlled by the bulk magnetic field. We discuss the stability limit of these droplets, where they depin from the chemical inhomogeneity and grow beyond it, causing a fast phase transformation. In Sec. V, we analyze the dynamics of nucleation events, characterizing both the growth process of a single nucleus from nanoscopic to macroscopic sizes and the distribution of nucleation times. Also, we compare these results to previous findings for single-droplet nucleation in the bulk. . $^{37,56,57}$ Finally, Sec. VI summarizes our conclusions. The extension of the classical theory of heterogeneous nucleation of Volmer and Turnbull ${ }^{9-12}$ to the two-dimensional case is given in Appendix A. In Appendix B, the depinning of droplets from a chemical heterogeneity in $d=3$ dimensions is briefly discussed.

\section{MODEL AND SIMULATION DETAILS}

The chosen model is similar to our previous work ${ }^{55}$ where a two-dimensional Ising/lattice gas model on the square lattice in $L \times M$ geometry was studied at phase coexistence (bulk field $H_{b}=0$ ). We apply periodic boundary conditions in the $x$-direction only, while free boundaries are used in the $y$-direction, and the Ising spins in the first $(i=1)$ and last $(i=L)$ rows experience boundary fields, cf. Fig. 1. At the upper boundary, we choose a homogeneous boundary field $H_{w 1}=-0.225$ throughout, which hence favors the minus spins, 
$S(i, j)=-1$; in lattice gas language, the local density variable $\rho(i, j)=(1+S(i, j)) / 2$ at a lattice site with coordinates $i(i=1$, $\ldots, L)$ in the $y$-direction and $j(j=1, \ldots, M)$ in the $x$-direction is then $\rho(i, j)=0$. At the lower boundary, $i=1$, we choose the boundary field inhomogeneous: from the site $j=(M-b) / 2+$ 1 to the site $j=(M+b) / 2$, we choose a positive boundary field $H_{w 3}=+0.90$, to favor along a line of length $b$ ( $b$ is a large odd integer) the liquid phase. Outside this region, the boundary field in the first row is $H_{w 2}=-0.90$ throughout, to ensure for the chosen total linear dimensions that in the absence of a bulk field $H_{b}$ the vapor phase (or phase with negative magnetization, respectively) is the stable phase, for all choices of $b$ that were considered.

The temperature $T$ is measured in units of the critical temperature $T_{c b}$ of the bulk; i.e., ${ }^{48} k_{B} T_{c b} / J=2 / \ln (1+\sqrt{2}) \approx$ 2.27, where $k_{B}$ is Boltzmann's constant and $J$ is the exchange constant. Boundary and bulk fields $H_{w 1}, H_{w 2}, H_{w 3}, H_{b}$ are measured in units of $J$. Thus, the used Hamiltonian is

$$
\begin{aligned}
\mathcal{H}= & -J \sum_{i=1}^{L} \sum_{j=1}^{M} S(i, j)[S(i+1, j)+S(i-1, j)+S(i, j+1) \\
& +S(i, j-1)] / 2-H_{w 1} \sum_{j=1}^{M} S(L, j)-\sum_{j=1}^{M} H_{w}(j) S(1, j) \\
& -H_{b} \sum_{i=1}^{L} \sum_{j=1}^{M} S(i, j), \quad S(i, j)= \pm 1
\end{aligned}
$$

where $H_{w}(j)=H_{w 2}$ for $1 \leq j \leq(M-b) / 2$ and $(M+b) / 2+1 \leq$ $j \leq M$, while $H_{w}(j)=H_{w 3}$ for $(M-b) / 2+1 \leq j \leq(M+b) / 2$. Also, $S(i, j)=0$ is taken for missing neighbors.

The chosen value of $H_{w 3}=0.90$ leads to a wetting critical temperature $t_{w}=T_{w} / T_{c b} \simeq 0.4866 .{ }^{50}$ Furthermore, heterogeneous nucleation is studied for $T<T_{w}$ so that the order within the bulk domains is almost perfect, and the correlation length in the bulk is of the order of the lattice spacing. For all temperatures $T<T_{w}$, we have a nonzero contact angle $\theta_{c}<90^{\circ}$ in the region where $H_{w 3}$ acts, while due to the antisymmetric choice $H_{w 2}=-H_{w 3}$ the contact angle is $\pi-\theta_{c}$ in the region where $H_{w 2}$ acts.

For all simulations, we choose $M=453$ and $L=300$ to make sure that there are no finite size effects associated with interfacial fluctuations. Also, in order to make it easier for the reader to establish the connection to fluid droplets, we will describe all our results in terms of local densities defined via $\rho(i, j)=(\langle S(i, j)\rangle+1) / 2$. Monte Carlo simulations were carried out with standard single spin-flip algorithms, ${ }^{43}$ which, as is well known, ${ }^{43}$ can be interpreted as a simulation of a stochastic process, where (in a lattice gas terminology) particles are randomly adsorbed at, or desorbed from, the sites of the lattice; the rates of these processes satisfy the detailed balance condition with the Hamiltonian, Eq. (1). Note that the density in the considered lattice gas is not a conserved variable, of course; hence, the physical situation that is simulated is a two-dimensional substrate in equilibrium with an ideal gas reservoir, at the specified temperature and chemical potential, corresponding to the chosen bulk magnetic field. Of course, the Monte Carlo process has no intrinsic time units for the rates of Monte Carlo moves: so time is measured in units of “attempted Monte Carlo steps per spin (MCS)" rather than any physical time units. Simulations are started with an initial condition where all spins are taken as $S(i, j)=-1$, i.e., $\rho(i, j)=0$ in accord with the nonwet ground state of the system. Then, the system is equilibrated up to $5 \times 10^{6}$ MCS by taking $H_{b}=0$ and subsequently is "quenched" to $H_{b}>0$ in order to observe nucleation. Runs are performed for additional $2 \times 10^{7}$ MCS, and averages are taken after disregarding $1 \times 10^{7}$ MCS.

\section{WALL ATTACHED PRECURSOR DROPLETS IN METASTABLE EQUILIBRIUM}

In our system, at least in the limit $M \rightarrow \infty, L \rightarrow \infty$, the vapor phase is the true equilibrium phase only for $H_{b} \leq 0$. But when we consider a situation where equilibrium in the bulk has been established for $H_{b}=0$, and at the time of the Monte Carlo sampling that we take as the origin of time $(\tau=0)$, the field $H_{b}$ is instantaneously switched to a small positive value, the vapor phase in the bulk may reach a state of metastable equilibrium, with a "lifetime" larger than the observation time $\tau_{o b s}$ of the simulation. This metastability can be already understood qualitatively by the classical theory of heterogeneous nucleation from the work of Volmer and Turnbull; ${ }^{9-12}$ see Appendix A.

In this region where the time $\tau_{N}$ to observe nucleation satisfies the condition $\tau_{N} \gg \tau_{o b s}$, one may observe the formation of wall-attached precursor droplets in the part of the sample where the surface field $H_{w 3}$ acts, Fig. 1. Note that we restrict attention to temperatures $T$ distinctly lower than $T_{w}$, i.e., when partial wetting of the wall occurs. To avoid confusion, we stress that we denote the boundaries at $i=1$ and $i=L$ in our system as "walls" although they are onedimensional lines only, Fig. 1. If we would choose $T>T_{w}$, i.e., the case of complete wetting of the wall for $b \rightarrow \infty$, there would no longer occur any nucleation barrier; the liquid wetting layer that occurs then at the wall already for $H_{b}=0$ would immediately grow by increasing its thickness as soon as $H_{b}>0$. For finite $b$, the interface of the liquid droplet is still pinned to the walls near the sites where the boundary field changes from $H_{w 3}$ to $H_{w 2}$. In our previous work, ${ }^{55}$ we have verified a prediction of Jakubczyk et al. ${ }^{58,59}$ for the case $H_{b}=0$ and $T>T_{w}$, based on the solid-on-solid (SOS) model in terms of the interface Hamiltonian approach for the excess density $\Delta \rho$ due to the droplet, given by

$$
\Delta \rho=\left(\rho_{\ell}^{\text {coex }}-\rho_{v}^{\text {coex }}\right) \frac{1}{4} b^{3 / 2} \sqrt{\pi / 2 \Sigma(T)}, \quad b \rightarrow \infty .
$$

In Eq. (2), $\rho_{\ell}^{\text {coex }}$ and $\rho_{v}^{\text {coex }}$ are the coexisting liquid and vapor densities in the bulk, respectively. Note that $\rho_{\ell}^{\text {coex }}-\rho_{v}^{\text {coex }}$ $=m^{\text {coex }}$, the spontaneous magnetization of the Ising model. ${ }^{49}$ Also, $\Sigma(T)$ is the interfacial stiffness of the Ising model. ${ }^{60}$ Equation (2) implies that the average droplet shape is a semiellipse, with a small axis proportional to $b^{1 / 2}$ for $b \rightarrow \infty$. For large $b$, even this small axis can easily exceed the critical droplet radius $R^{*}$ for homogeneous nucleation in the bulk (see Appendix A), and thus, it is plausible that for large but finite $b$ the nucleation barrier will be very small. Although a study of the phase transformation for $T>T_{w}$ and not so large $b$ may be interesting in its own right, we here fixed the attention 

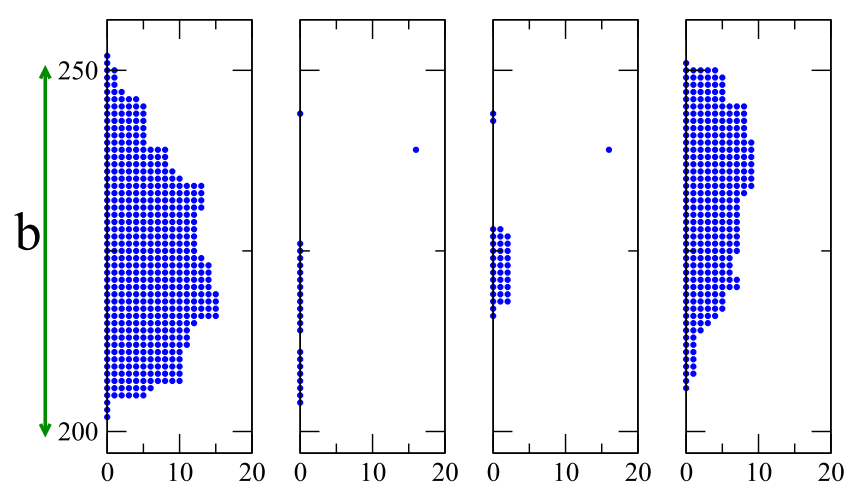

FIG. 2. Snapshot configurations of the heterogeneous nucleation of a droplet in an external field $\left(H_{b}\right)$. Note that unlike Fig. 1, the boundaries are oriented along the ordinate direction and the $y$-coordinate along the abscissa. Data obtained for $t=0.40$ and $b=51$ (note the double arrow in the left-hand side of the figure indicating the length $b$ of the heterogeneity). Each snapshot corresponds to different realizations obtained with the same parameters but using different seeds in the random number generator. The simulated systems have size $L=300$ and $M=453$, while the snapshots only show the central part, horizontal (vertical) length 20 lattice units (60 lattice units), where the droplets develop. Note that the same length scale has been used for both sides of the panels in order to display the actual shape of the droplets. The system was equilibrated at $H_{b}=0$ during $5 \times 10^{6} \mathrm{MCS}$, subsequently "quenched" to $H_{b}=0.030$, and all the snapshots are obtained for $\tau=2 \times 10^{7} \mathrm{MCS}$.

to $T<T_{w}$, where the linear dimension in the $y$-direction of the wall-attached droplet for $H_{b}=0$ remains finite for $b \rightarrow \infty$. We have also shown ${ }^{55}$ that the density in the mid-point $j_{\text {half }}$ $=(M+1) / 2$ of the inhomogeneity of the boundary is compatible with an exponential decay with the distance $y$ from the wall ( $y$ is only defined at the discrete lattice indices $i$, of course),

$$
\rho\left(i, j_{\text {half }}\right)=A_{0}(t) \exp \left(-y / \xi_{\perp}(b, t)\right), \quad H_{b}=0 .
$$

Note that in the context of various theoretical concepts on interfaces in this constrained geometry, the use of a continuum description (in terms of coordinates $x, y$ ) is mandatory; but for a precise characterization of the simulation setup on the lattice, discrete indices of the lattice points $(j$ in the $x$-direction, $i$ in the $y$-direction) have also to be used. Here, $A_{0}(t)$ is an amplitude factor, $t=T / T_{c b}$ must be less than $t_{w}=T_{w} / T_{c b}$, and the decay length $\xi_{\perp}(b, t)$ converges to the standard transverse correlation length $\xi_{\perp}(t)$ familiar from the theory of critical wetting ${ }^{51}$ in $d=2$ dimensions, with $\xi_{\perp}(t) \propto\left(t_{w}-t\right)^{-1}$. In order to avoid critical fluctuations associated with the second-order wetting transition, we consider here only temperatures distinctly lower than $t_{w}$, namely, $t=0.30,0.35$, and 0.40 (recall that $t_{w}=0.4866$ for our choice of $h_{w 3}{ }^{50,51}$ ).

When we now consider metastable phases with small but nonzero $H_{b}>0$, we find that the actual droplet configurations are strongly fluctuating (Fig. 2) even though the average profile $\rho\left(i, j=j_{\text {half }}\right)$ is a smooth function [Fig. 3(a)]. We note that for small enough $H_{b}$ (such as $H_{b} \leq 0.02$ ) the decay of the profile with the distance $y$ from the boundary is still compatible with Eq. (3), and both the amplitude $A_{0}(t)$ and the decay length $\xi_{\perp}(b$, $t$ ) increase with $H_{b}$ only very slowly. However, when we reach the apparent limit of metastability $H_{b}^{\max }$, where for the chosen observation time $\tau_{o b s}=2 \times 10^{7}$ MCS nucleation becomes observable, which in the case of Fig. 3(a) is $H_{b}^{\max } \approx 0.033$, the behavior changes: In the regime $0.02 \leq H_{b} \leq 0.032$, the profiles change from a simple exponential decay to sigmoidal, and $A_{0}(t)$ moves towards $\rho_{\ell}^{\text {coex }}$, which on the scale of Fig. 3(a) is indistinguishable from unity, while $\rho_{v}^{\text {coex }}$ is not distinguishable from zero here. Thus we have defined an effective droplet height $h_{\text {eff }}\left(t, H_{b}\right)$ by measuring the distance $y$ of the inflection point from the coordinate origin [Fig. 3(b)]. The increase of $h_{\text {eff }}\left(t, H_{b}\right)$ with $H_{b}$ is clearly faster than a straight line through the origin. An intriguing feature is the fact that the ratio $h_{\text {eff }}\left(t, H_{b}\right) / b$ seems to be a function of the scaling combination $b H_{b}$ only [Fig. 3(c)].
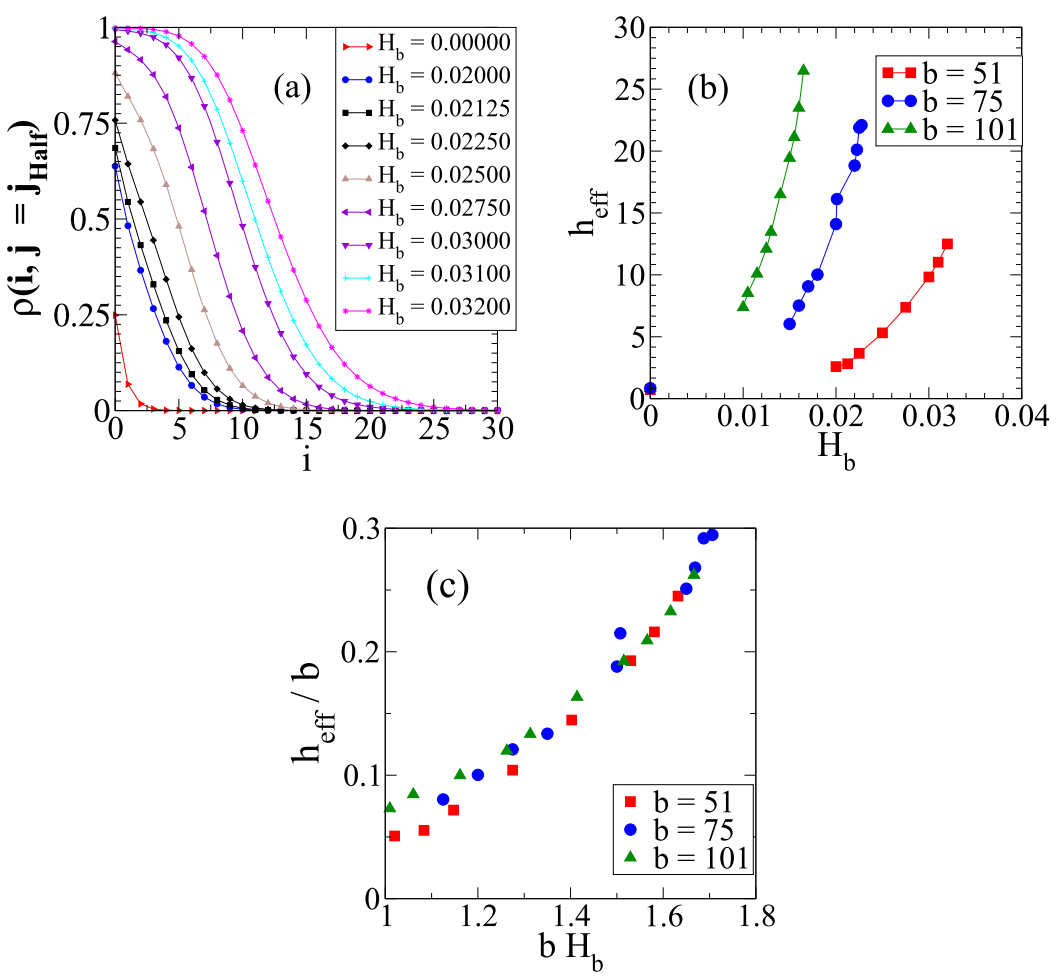

FIG. 3. (a) Plots of the density profiles measured at the center of the sample $\left(\rho\left(i, j=j_{\text {Half }}\right)\right)$ versus the distance to the wall $y$, here $j_{\text {Half }}=\left(\frac{M}{2}+1\right)=227$. Of course, on the lattice, only discrete distances $y=1,2,3, \ldots$ are possible, so the continuous curves are guides to the eye only. Data corresponding to $t=0.40, b=51$, and different values of the bulk field, as indicated. (b) Plot of the effective height $\left(h_{e f f}\right)$ of the droplet as estimated by measuring the distance from the wall $(i=1)$ to the inflection point of the density profile. Symbols at $H_{b}=0$ correspond to the decay length $\xi_{\perp}(b, t)$ obtained by fitting the profiles with the aid of Eq. (3), ${ }^{55}$ namely, $\xi_{\perp}(101,0.40)=0.8506$, $\xi_{\perp}(75,0.40)=0.8379$, and $\xi_{\perp}(51,0.40)=0.7338$. Data obtained for $t=0.40$ and three choices of $b$, as indicated. The rightmost point for each choice of $b$ corresponds to the value $H_{b}=H_{b}^{\max }$, since for slightly larger choices of $H_{b}$ no metastable droplets could be observed. (c) Scaling plot of $h_{\text {eff }} / b$ versus $b H_{b}$ as obtained with the data already shown in panel (b). 


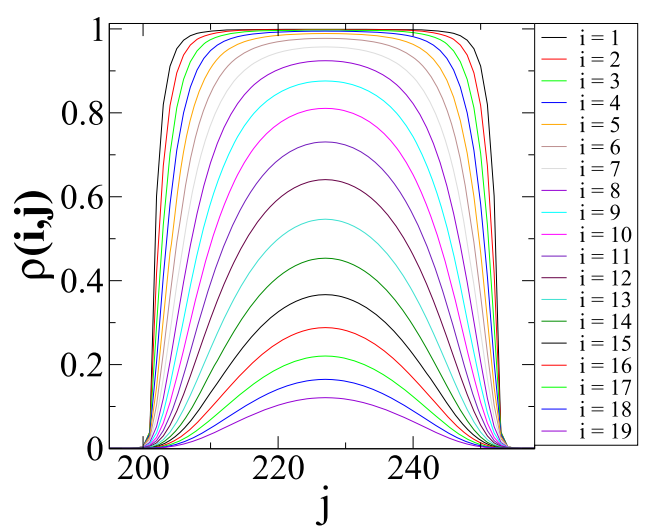

FIG. 4. Density profiles $\rho(i, j)$ measured in the $x$-direction parallel to the wall and for different values of the distance $i$, perpendicular to the wall, as indicated. Data correspond to $b=51, t=0.40$, and $H_{b}=0.032$.

In order to characterize the droplet shape more precisely, also density profiles $\rho(i, j)$ in the $x$-direction parallel to the boundary were taken (Fig. 4). It is clear that in the shown example the droplet has considerable extent in the $x$-direction, comparable to $b$, as long as the distance $i$ from the boundary is clearly less than $h_{\text {eff }}$. Thus we have introduced a characteristic length of the wall-attached droplet in the $x$-direction, defining its "baseline" $b_{\text {eff }}$ as the area below the density profile for $i=1$, namely,

$$
b_{\text {eff }}=\sum_{j=(M-b) / 2+1}^{(M+b) / 2} \rho(i=1, j) .
$$

Figure 5(a) shows a plot of $b_{\text {eff }} / b$ for three choices of $b$ as a function of $H_{b}$. One can see that for $H_{b} \ll H_{b}^{\max } b_{\text {eff }} / b$ is small but saturates at unity when $H_{b}$ approaches $H_{b}^{\max }$. Again the data for the three choices of $b$ almost superimpose on a master curve when one plots $b_{\text {eff }} / b$ as a function of the product $b H_{b}$ [Fig. 5(b)]; we shall discuss this scaling behavior of the characteristic lengths $h_{\text {eff }}$ and $b_{\text {eff }}$ below.

When the ratio $b_{\text {eff }} / b$ is no longer small, one can still use density profiles such as those shown in Fig. 4 to evaluate the effective length of the droplet parallel to the boundary but evaluated at a distance $i>1$ from the wall [e.g., by performing the corresponding summation of Eq. (4)] and characterize the average shape of the droplet (note that this procedure is
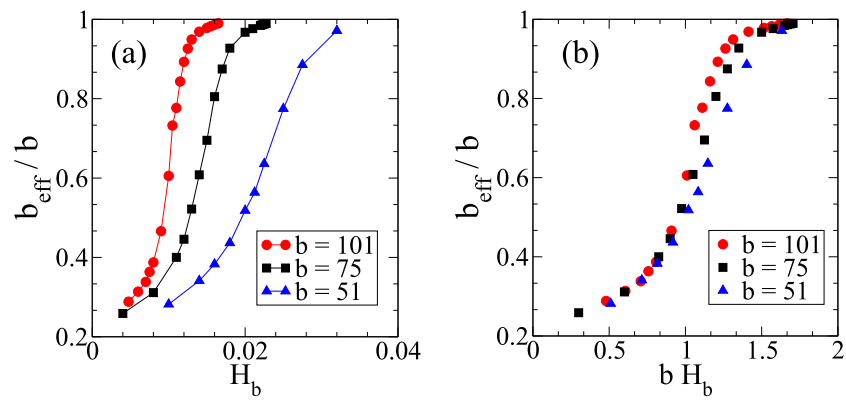

FIG. 5. (a) Plots of the normalized effective length of the droplet in contact with the wall (i.e., the "baseline") $\left(b_{\text {eff }} / b\right)$ versus $H_{b}$, as measured for the regime of wall-attached metastable droplets (precursor of the actual nucleation that takes place for $H_{b}>H_{b}^{\max }$ ). Results obtained for $t=0.40$ and different values of $b$, as indicated. (b) Scaling plot $b_{\text {eff }} / b$ versus $b H_{b}$ of the data already shown in panel (a).

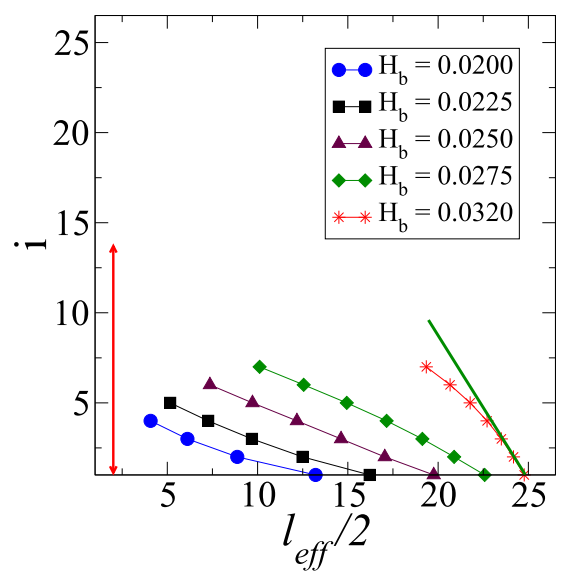

FIG. 6. Plot of the effective (half) length of the droplets $\left(l_{e f f} / 2\right)$ as obtained from the integration of the density profiles as shown in Fig. 4 (horizontal axis) versus the distance to the wall where the inhomogeneity is placed (vertical axis). Data obtained for $t=0.40, b=51$, and different values of the bulk field, as indicated. The double arrow at the left-hand side of the figure shows the effective height of the droplet $\left(h_{\text {eff }}\right)$ as measured for $H_{b}=0.032$ [see Fig. 3(b)]. The full straight line at the right-hand side of the figure shows the asymptotic slope of the droplet contour that is used to determine the effective contact angle $\theta_{\text {eff }}$ (see also Fig. 7).

equivalent to the construction of contours of constant density); see Fig. 6. The slope of these contours can be used to extract estimates for the effective contact angle $\theta_{\text {eff }}\left(H_{b}\right)$ from the part of the contours at small $i$ values, where these contours representing the coarse grained interface positions reach the wall. Figure 6 exploits this idea for the case $t=0.40, b=51$, and several choices of $H_{b}$. By fitting straight lines to these contours in the region close to the wall (i.e., for $i=1,2,3$ ), estimates of $\theta_{\text {eff }}\left(H_{b}\right)$ can be extracted [Fig. 7(a)]. It is seen that for choices of $H_{b}$ for which $b_{\text {eff }}\left(H_{b}\right)$ is distinctly smaller than $b, \theta_{\text {eff }}\left(H_{b}\right)$ is essentially independent of $H_{b}$, and of the order of $\theta_{\text {eff }} \approx 10^{\circ} \pm 2^{\circ}$ in this case. However, when $b_{\text {eff }}\left(H_{b}\right)$ starts to saturate at $b$, the ratio $h_{\text {eff }} / b$ and the effective contact angle $\theta_{\text {eff }}$ both start to increase rather distinctly. As Fig. 7(b) demonstrates, this increase of $\theta_{\text {eff }}$ starts at $b H_{b} \approx 1$. Metastable precursor droplets are found up to angles of $\theta_{\text {eff }}^{\max } \approx 70^{\circ}$ when $H_{b}$ reaches $H_{b}^{\max }$.

A related conclusion can be drawn with even less ambiguity, since it does not require an analysis of the shape of the
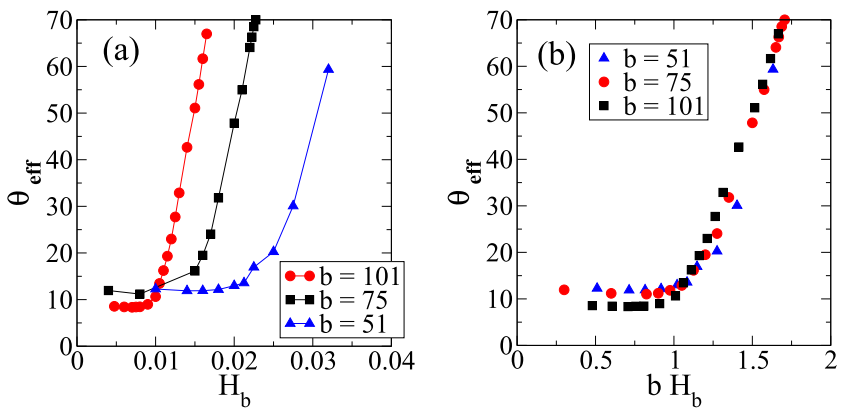

FIG. 7. (a) Plots of the effective contact angle of the droplets $\left(\theta_{\text {eff }}\right)$ versus $H_{b}$, as measured for the regime of metastable wall-attached droplets (precursor of the actual nucleation) that takes place for $H_{b}<H_{b}^{\max }$, where $H_{b}^{\max }$ is the value of the field where the transition to the liquid phase covering the whole sample is observed in the simulations. Results obtained for $t=0.40$ and different values of $b$, as indicated. (b) Scaling plot $\theta_{\text {eff }}$ versus $b H_{b}$ of the data already shown in panel (a). 

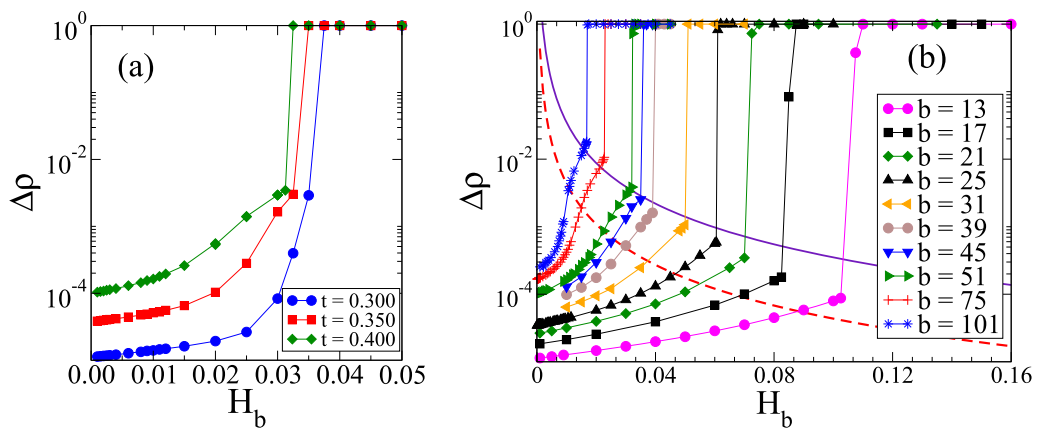

FIG. 8. (a) Log-linear plot of the total density excess $\Delta \rho$ plotted versus $H_{b}$ for three choices of $t$, as indicated. Data obtained for $b=51$. (b) Log-linear plot of $\Delta \rho$ versus $H_{b}$ obtained at temperature $t=0.40$ and different values of $b$, as indicated. The dashed (full) curve is a plot of $R^{*^{2}} \pi f_{V T} / L M$ [see Eq. (5) noting $\Delta \rho=\Delta m /(L M)$ ] versus $H_{b}$ as obtained by assuming $\theta_{c}=34.155^{\circ}\left(\theta_{c}=\theta_{e f f}^{\max }\right.$ $=70^{\circ}$ ) in the evaluation of $f_{V T}$. More details in the text. precursor droplet, when we simply record the excess density $\Delta \rho$ in the system due to the droplet (Fig. 8). In fact, Fig. 8(a) shows plots of $\Delta \rho$ versus $H_{b}$ at three choices of $t$ for $b=51$, while Fig. 8(b) shows plots of $\Delta \rho$ versus $H_{b}$ for $t=0.40$ and various choices of $b$, as indicated. Note that in both cases the states where the transition to the liquid in the simulated system has occurred are included, namely, all the data points with $\Delta \rho=1$ implying that the whole simulation box is filled uniformly by a liquid. The excess density of the precursor droplet in these plots is normalized by dividing the "excess mass" $\Delta m$ contained in the precursor droplet by the total number of lattice sites, $N=L \times M=300 \times 453=135900$. Recall that the excess density is the difference between the density measured in the presence of the inhomogeneity in the wall and that obtained for an homogeneous wall. Figure 8(a) implies that the value of $\Delta m$ where the precursor droplet is large enough to trigger nucleation of the liquid phase in the system depends only weakly on temperature; it is $\Delta m \approx 400$ for $b=51$, and the choice $H_{w 2}=0.90$ that was made here. But again there is a pronounced dependence of $\Delta \rho$ (or $\Delta m$, respectively) at the transition on the choice of $b$ [Fig. 8(b)].

At this point, it is interesting to make contact with the classical theory of heterogeneous nucleation as formulated in $d=3$ dimensions by Volmer and Turnbull. ${ }^{9-12}$ A simple adaptation of this theory to $d=2$ (see Appendix A) implies that a critical droplet on a homogeneous substrate with contact angle $\theta_{c}$ involves an excess density mass given by

$$
\Delta m=\left(\rho_{\ell}^{\text {coex }}-\rho_{v}^{\text {coex }}\right)\left(R^{*}\right)^{2} \pi f_{V T}\left(\theta_{c}\right) .
$$

Here, $R^{*}$ is the critical radius of homogeneous nucleation, which in the classical theory simply is

$$
R^{*}=\frac{f_{\text {int }}}{2\left(\rho_{\ell}^{\text {coex }}-\rho_{v}^{\text {coex }}\right) H_{b}},
$$

with $f_{\text {int }}$ being the interfacial tension between coexisting bulk phases separated by a flat interface. As discussed in
Appendix A, Eqs. (5) and (6) ignore the anisotropy of the interfacial tension, assuming a circular droplet in the bulk, and a circle cut shape of the droplet at the boundary, where the coarse-grained interface makes an angle $\theta_{c}$. The VolmerTurnbull function $f_{V T}\left(\theta_{c}\right)$ measures the reduction of the droplet area of the circle cut relative to the full circle and in this approximation (see Appendix A) is given by

$$
f_{V T}(\theta)=\frac{1}{\pi}\left(\theta-\frac{\sin (2 \theta)}{2}\right)
$$

The upper curve in Fig. 8(b) shows $\Delta \rho=\Delta m / L M$ as obtained according to Eqs. (5)-(7) by using the "observed" contact angle $\theta_{c}=\theta_{\text {eff }}^{\max } \approx 70^{\circ}$ just at the transition to the liquid phase. However, by using a theoretical estimate of the contact angle in thermal equilibrium obtained by means of a SOS approximation $\theta_{c}$ due to Abraham et al. ${ }^{52}$ would imply $\theta_{c} \approx 34^{\circ}$, predicting hence distinctly smaller critical droplets [Fig. 8(b)]. However, for both choices of $\theta_{c}$, our results (Figs. 5 and 7) imply that $b_{\text {eff }}$ is of the same order as $b$ when nucleation occurs. Since the baseline length $b_{d r o p}^{*}$ of the circle cut critical droplet satisfies the geometrical relationship

$$
b_{d r o p}^{*}=2 R^{*} \sin \left(\theta_{c}\right) \text {, }
$$

we can eliminate $R^{*}$ in Eqs. (5) and (6) in favor of $b_{d r o p}^{*}=b$, and in this way, the two theoretical curves in Fig. 8(b) were obtained. Figure 9(a) shows then the scaling plot of $\Delta \rho / b^{2}$ versus $b H_{b}$, validating the idea that $\Delta m$ scales like $b^{2}$ and is a function of the product $b H_{b}$, as $b_{\text {eff }}$ and $h_{\text {eff }}$ [Figs. 3(c) and 5(b)]. Both Figs. 7 and 8 show that two regimes exist: for $b H_{b}$ $\lesssim 1, \theta_{\text {eff }}$ is very small, $l_{\text {eff }}<b$ (Fig. 8), $\Delta \rho$ increases slowly with $b H_{b}$, and the droplet density profile [Fig. 3(a)] decays with the distance from the inhomogeneity like an exponential. All these properties change for $b H_{b} \gtrsim 1$. Note that Fig. 9(a) includes both the regime $b H_{b} \lesssim 1$, where scaling is not expected hold, and the true scaling regime $b H_{b} \gtrsim 1$. Of course, for $H_{b}=0$ in the nonwet regime, the excess density $\Delta \rho$ only scales like $\Delta \rho \propto b$;
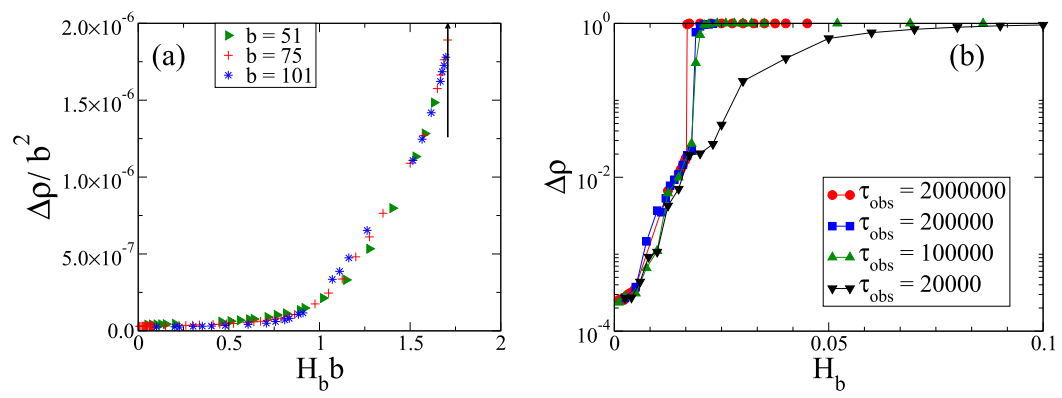

FIG. 9. (a) Scaling plot of the total density excess, $\Delta \rho / b^{2}$ versus $b H_{b}$, according to Eq. (17). Data taken from Fig. 8(b). The vertical arrow at the right-hand side of the figure indicates the asymptotic value $b H_{b}^{\max } \simeq 1.71$ for the observation of nucleation, which yields $\theta_{c}^{\max } \approx 70^{\circ}$ as it follows from Eqs. (6) and (8). (b) Log-linear plot of the total density excess, $\Delta \rho$ plotted versus $H_{b}$, for the case $t=0.40, b=51$, and 4 choices of the observation time $\tau_{o b s}$ of the metastable state, as indicated. 
thus in the regime of small $b H_{b}$, a crossover from $\Delta \rho \propto b$ to $\Delta \rho \propto b^{2}$ with increasing $b H_{b}$ must occur.

In order to give a physical interpretation of the simple scaling of all quantities ( $b_{\text {eff }}, h_{\text {eff }}$, and $\Delta \rho$ ) with the product $b H_{b}$, we recall the description of droplets at chemical inhomogeneous substrates in $d=2$ dimensions in terms of the interface Hamiltonian proposed by Jakubczyk et al. ${ }^{58,59}$ In this description in the spirit of a solid-on-solid (SOS) model, the problem is described by a one-dimensional degree of freedom, namely, the distance $y=\ell(x)$ of the (locally sharp) interface from the boundary at $y=0$ (involving a continuum approximation). So the effective (coarse-grained) Hamiltonian is, absorbing a factor $\frac{1}{k_{B} T}$ here,

$$
\mathcal{H}[\ell(x)]=\int_{-M / 2}^{+M / 2} d x\left[\frac{\Sigma(T)}{2}\left(\frac{d \ell}{d x}\right)^{2}+V(x, \ell)\right],
$$

where both fluctuations in the bulk and overhangs of the interface are neglected, with $V(x, \ell)$ being the effective potential acting on the interface. Recall that in the SOS treatment the interfacial stiffness $\Sigma(T)^{60}$ of the one-dimensional interface is considered, instead of the actual interfacial tension $f_{\text {int }}$ of the Ising model. ${ }^{48}$ For the considered situation, for $|x|>b / 2$ the boundary at $y=0$ strongly favors the vapor phase, so we have essentially $\ell(x)=0$ there, as one can verify from Fig. 4. So, Eq. (9) can be reduced to

$$
\mathcal{H}[\ell(x)]=-\int_{-b / 2}^{+b / 2} d x\left[\frac{\Sigma(T)}{2}\left(\frac{d \ell}{d x}\right)^{2}+V(x, \ell)\right] .
$$

Note that Eqs. (9) and (10) also assume $\left|\frac{d \ell}{d x}\right| \ll 1$ everywhere, an assumption that is somewhat questionable in view of the actual snapshots of the interfacial configurations (Fig. 2), at least near $x= \pm \frac{b}{2}$; however, since no actual calculations on the basis of Eqs. (9) and (10) are done here, this problem does not matter.

Now the effective potential $V(x, \ell)$ can be written as

$$
V(x, \ell)=\left[V_{0}(x, \ell)-\ell\left(\rho_{\ell}^{\text {coex }}-\rho_{v}^{\text {coex }}\right) H_{b}\right] / k_{B} T,
$$

where $V_{0}(x, \ell)$ is the potential binding the interface to the wall for $H_{b}=0$. Only this latter case has been considered by Jakubczyk et al. ${ }^{58,59}$ Applying a field $H_{b}>0$ favors the liquid phase, and thus the potential decreases proportional to $\ell H_{b}$.

Now the key observation is that the dependence on $b$ is elucidated when we rescale all distances by $b$, namely,

$$
x=b x^{\prime}, \quad \ell=b \ell^{\prime},
$$

which yields $\mathcal{H}=b \mathcal{H}^{\prime}$ with

$$
\mathcal{H}^{\prime}\left[\ell^{\prime}\left(x^{\prime}\right)\right]=-\int_{-1}^{+1} d x^{\prime}\left[\frac{\Sigma(T)}{2}\left(\frac{d \ell^{\prime}}{d x^{\prime}}\right)^{2}+V\left(x^{\prime}, \ell^{\prime}\right)\right],
$$

with

$$
V\left(x^{\prime}, \ell^{\prime}\right)=V_{0}\left(x^{\prime}, \ell^{\prime}\right)-\ell^{\prime}\left(\rho_{\ell}^{\text {coex }}-\rho_{v}^{\text {coex }}\right) b H_{b} .
$$

Now the partition function needs to be evaluated as a path integral,

$$
\mathcal{Z}=\int \mathcal{D} \ell^{\prime} \exp \left(-b \mathcal{H}^{\prime}\right)
$$

and from Eqs. (13) and (14), we conclude that the boundary excess free energy $\Delta F=-k_{B} T \ln (\mathcal{Z})$ due to the droplet depends on the variables $b, t$, and $H_{b}$ in the following scaled form, $f\left(t, b H_{b}\right)$ being the free energy density per length unit along the boundary:

$$
\Delta F=b f\left(t, b H_{b}\right)
$$

The excess density due to the droplet is obtained from Eq. (16) via a derivative with respect to $H_{b}$, i.e.,

$$
\Delta \rho=b^{2} \tilde{M}\left(t, b H_{b}\right),
$$

where $\tilde{M}$ is the resulting scaling function of the excess mass. Equation (17) hence justifies the choice of scaling variables for Fig. 9(a).

This scaling property is subtle, of course, due to the requirement of metastable equilibrium: it is implied also by Eq. (11) that there cannot be for $H_{b}>0$ a true equilibrium at any finite value of $\ell$, so Eq. (15) makes sense only for a suitably constrained partition function.

So the droplets studied so far can only be found in a suitable "window" of observation times $\tau_{o b s}$. In fact, $\tau_{o b s}$ must be large enough to allow that the wall-attached droplet reaches local equilibrium in spite of the slow and sluggish fluctuations of the interface configuration (Fig. 2). But at the same time, $\tau_{o b s}$ must be small enough that nucleation events (where the droplet grows fast to the full size of the system, see Sec. V) are still negligible.

This consideration is exemplified in Fig. 9(b): here a $\log -\log$ plot of the excess density in the system versus $H_{b}$ is shown, for four choices of $\tau_{o b s}$. For very small $H_{b}$, such as $H_{b}=0.0025$, the dependence on $\tau_{o b s}$ is negligible since the time $\tau_{N}$ needed to nucleate is astronomically large, and the wall attached droplet is very tightly bound to the wall [cf. Fig. 3(a)], so it is rather easily equilibrated. However, for $H_{b}=0.01$ we see that data for $\tau_{o b s}$ $=2 \times 10^{5}$ and $\tau_{o b s}=2 \times 10^{6}$ perfectly agree and nucleation is not yet possible; but the result for $\tau_{o b s}=2 \times 10^{4}$ is clearly smaller, this observation time was insufficient to sample fluctuations such as those seen in Fig. 2 exhaustively. For $H_{b}=0.018$ however, there is also a systematic difference between $\tau_{o b s}=2 \times 10^{5}$ and $\tau_{o b s}=2 \times 10^{6}$ : for the latter time, nucleation typically has occurred, while for the former time, the metastable boundary-attached droplet is still visible.

Since Fig. 5 suggests that we can (for the choices of $b$ used here) observe metastable boundary attached droplets up to $b_{\text {eff }}$ $\approx b$, we have also tested as a possible hypothesis that these boundary-attached droplets with $b_{\text {eff }}=b$ can be described by the Volmer-Turnbull theory of heterogeneous nucleation (see Appendix A). This theory assumes that the critical droplet causing nucleation is a cut from a sphere (circle in our $d=2$ case) with radius $R^{*}$ [Eq. (6)], the angle of the sphere cut with the straight line representing the boundary being the contact angle $\theta_{c}$. Geometry then implies $b_{\text {eff }}=b$ as quoted in Eq. (8), and combining Eqs. (6) and (8) yields a relationship between $b$ and $H_{b}$,

$$
b_{\text {drop }}^{*}=b=f_{\text {int }} \sin \left(\theta_{c}\right) /\left(\rho_{\ell}^{\text {coex }}-\rho_{v}^{\text {coex }}\right) H_{b}^{\text {crit }},
$$

where $H_{b}^{c r i t}$ is the prediction of the standard theory of heterogeneous nucleation for the critical field at the onset of nucleation 


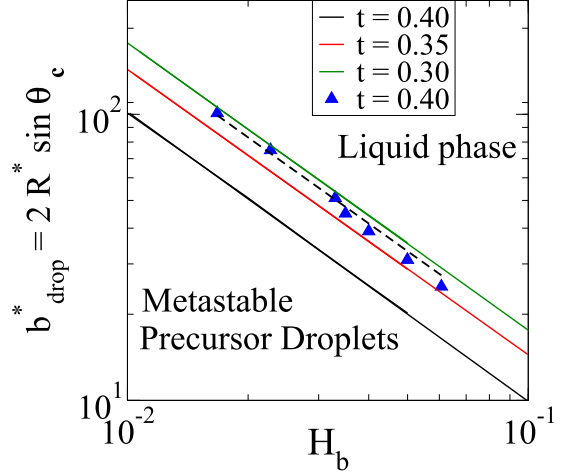

FIG. 10. Log-log plot of $b_{d r o p}^{*}=2 R^{*} \sin \left(\theta_{c}\right)$ versus $H_{b}$ corresponding to different temperatures as indicated. Full lines correspond to the theoretical prediction [Eq. (18)] that follows by considering the values of $\theta_{c}$ given by a SOS calculation [Eq. (19)]. The straight lines have slope -1 . Numerical data corresponding to $t=0.40$ are shown by full triangles. The dashed line is a fit of the numerical data, obtained by assuming a slope -1 , which yields $\theta_{c}^{\max }=\theta_{\text {eff }}^{\max }=67.5^{\circ}$ if Eq. (18) is invoked for the numerical simulation data. At each temperature, the full straight lines are the theoretical curves where nucleation according to the Volmer-Turnbull theory is predicted to occur when the baseline of the critical droplet equals $b$ so that they are the boundary between the region where metastable precursor droplets are observed (lower left-hand side of the panel) and the liquid phase (upper right-hand side of the panel). More details in the text.

of the liquid phase when the length of the baseline of the droplet is $b_{d r o p}^{*}=b$. This result is plotted in Fig. 10, using for $f_{\text {int }}$ the Onsager result ${ }^{48}$ for the interface tension of a straight interface oriented perpendicular to the lattice axis. For the contact angle $\theta_{c}$, we use the results derived from Abraham et al. ${ }^{52}$ in the SOS approximation, namely,

$$
\begin{aligned}
\tan \left[\theta_{c}\left(T_{w}, T\right)\right]= & \sinh \left[2\left(K-K_{w}\right] /[\cosh (K)\right. \\
& \left.-\cosh \left[2\left(K-K_{w}\right)\right]\right],
\end{aligned}
$$

where $K=J / k_{B} T$ and $K_{w}=J / k_{B} T_{w}$, respectively.

For our choice of $H_{w 3}$ implying $T_{w}=0.4866 T_{c b}$, Eq. (19) yields $\theta_{c} \approx 67,11^{\circ}, 50,05^{\circ}$, and $34,11^{\circ}$, for $t=T / T_{c b}=$ $0.30,0.35$, and 0.40 , respectively. The resulting straight lines in the log-log plot for $b$ versus $H_{b}$ [Eq. (18)] are compared to the estimates for the actual critical field $H_{b}^{\max }$ where the onset of nucleation of the liquid phase has been observed in the simulations [Fig. 10]. For each temperature, these lines correspond to the theoretical conditions where nucleation on the inhomogeneity of length $b$ becomes possible. The actual data points included in Fig. 10 separate the region of parameters where metastable precursor droplets are found and the liquid phase that already takes the whole simulation box. It is seen that the actual critical fields measured for $t=0.40$ always are larger than the predictions based on Eqs. (18) and (19). So, if we would fit the numerical data to Eq. (18), we would obtain the "observed" contact angle $\theta_{c}^{\max }=\theta_{\text {eff }}^{\max } \approx 67.5^{\circ}$, in excellent agreement with our previous estimations, e.g., showing that metastable precursor droplets are found up to angles of $\theta_{\text {eff }}^{\max } \approx 70^{\circ}$ [Fig. 7(b)], as well as from the scaling plot of Fig. 9 that also yields $\theta_{\text {eff }}^{\max } \approx 70^{\circ}$. The main reason for the difference between the field $H_{b}^{c r i t}$, predicted by the standard theory for heterogeneous nucleation and defined from Eq. (18) and calculated by using the contact angle $\theta_{c}$ obtained by means of the SOS approximation [Eq. (19)], and the actual critical field found in the simulations $H_{b}^{\max }$ is that in the regime $H_{b}^{c r i t}<H_{b}<H_{b}^{\max }$ the droplets nucleated at the inhomogeneity are pinned, see Sec. IV, i.e., their baseline cannot grow beyond $b$. However, it should be stressed that within this regime the area of the droplets actually grows by simultaneously increasing the contact angle and decreasing their radius. Only for $H_{b}>H_{b}^{\max }$ droplets "depin" and further growth is possible, with $b_{\text {drop }}>b$ and $\theta=\pi-\theta_{c}$; see also below. These pinned droplets should not be mistaken for the droplets described by the standard Volmer-Turnbull theory of heterogeneous nucleation, as discussed in Appendix A. In Sec. IV, we shall attempt a theoretical estimation of the field $H_{b}^{\max }$. We also note that for $H_{b}<H_{b}^{\text {crit }}$ only subcritical nuclei $\left(R<R^{*}\right)$ can form on the inhomogeneity, i.e., transient fluctuations occur whose average effect shows up in the exponentially decaying density profiles for $H_{b} \leq 0.022$ in Fig. 3(a).

Furthermore, it is worth discussing that Eq. (18) is not expected to be quantitatively accurate for several reasons: (i) The interface tension for a straight interface $f_{\text {int }}$ is used here, neglecting possible corrections due to the curvature of the droplet interface. (ii) Due to the anisotropy of the interfacial tension of the Ising lattice model, the actual shape of a large droplet of the liquid coexisting with surrounding vapor is not a circle; it rather resembles a square with rounded corners at low temperatures $^{61,62}$ (see also the largest droplet in Fig. 11, lefthand side panel). For heterogeneous nucleation, the droplet shape resulting from the appropriate Winterbottom construction $^{63}$ is then nontrivial to find, and the Volmer-Turnbull theory as presented in Appendix A needs to be extended to account
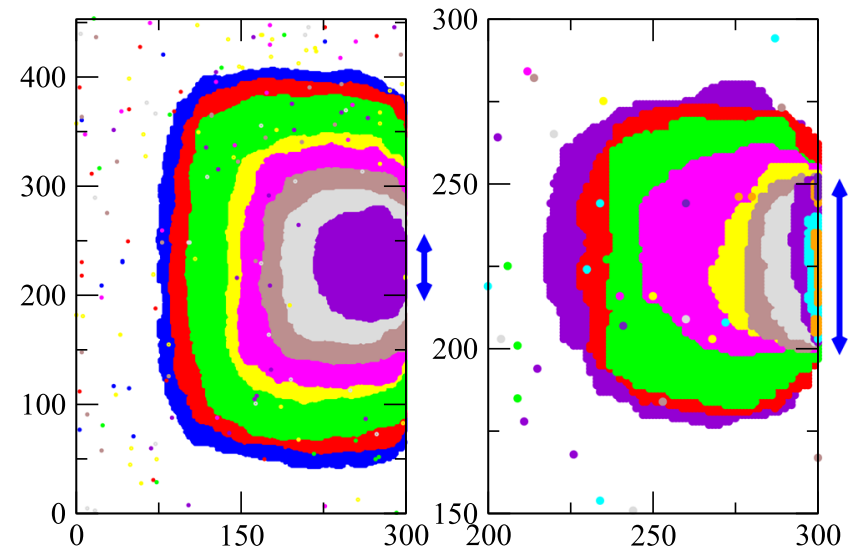

FIG. 11. Snapshot configurations of the heterogeneous nucleation of a droplet in an external field $\left(H_{b}\right)$. Data obtained for $t=0.40, b=51$ (note the double arrow in the right-hand side of each panel indicating the length of the heterogeneity), $L=300$, and $M=453$. The system was equilibrated at $H_{b}=0$ during $5 \times 10^{6} \mathrm{MCS}$ and subsequently "quenched" to $H_{b}=0.034$. The snapshot is recorded when the density of the nucleated droplet reaches the different values, which are identified by different colors. The simulation times $(\tau)$ in MCS necessary to reach each density are also listed between bracket. Then, going from high to low density, the values and colors are as follows: Panel (a) 0.556 [172 094] (blue), 0.493 [171 124] (red), 0.420 [169 948] (green), 0.260 [167 043] (yellow), 0.221 [166 148] (magenta), 0.153 [164 171] (brown), 0.100 [162 312] (grey), and 0.052 [159 035] (violet). Panel (b) 0.052 [159 035] [i.e., last value and color from panel (a)], 0.044 [158 518] (red), 0.037 [157 165] (green), 0.022 [152 678] (magenta), 0.010 [74 795] (yellow), 0.0075 [31 525] (brown), 0.0050 [12 212] (grey), 0.0025 [1773] (violet), 0.0010 [932] (cyan), 0.0008 [845] (magenta), and 0.0006 [787] (orange). Note the different scales for the abscissa and ordinate in both panels; while (a) shows the full system, (b) only shows a subpart of the system that contains the wall inhomogeneity plus attached droplet. More details in the text. 
for this anisotropy. For not very large droplets, also the "point" where the droplet interface meets the boundary can play a role, modifying Eq. (18) further, in analogy with the effect of the line tension of the sphere-cap shaped droplet on the contact angle in $d=3$ dimensions. ${ }^{64}$ In view of all these shortcomings of the existing theories, a more quantitative analysis of our numerical data for the boundary-attached droplets (Figs. 3-9) suffers from the incomplete knowledge of both the droplet shape and the equilibrium contact angle. Nevertheless, we attempt a phenomenological analysis of pinned droplets and their depinning in Sec. IV.

\section{DROPLETS PINNED AT CHEMICAL INHOMOGENEITIES AND THEIR “DEPINNING TRANSITION"}

When one deals with heterogeneous nucleation at homogeneous substrates, the nucleation barrier $\Delta F_{\text {het }}^{*}$ [Eq. (A13)] corresponding to the droplet having the critical radius $R^{*}$ [Eq. (6) or (A13), respectively] is all what matters: when such a droplet (of circle cut shape, with contact angle $\theta_{c}$ ) corresponding to the top of the free energy $\Delta F_{d r o p}(R)$ [Eq. (A12)] has been formed by a (rare) statistical fluctuation, with $50 \%$ probability this drop will grow with time $\tau$ after the nucleation event. For small fields $H_{b}$, the growth velocity is small, and then, we have "local equilibrium" of the growing droplet at the contact line; this means, at any instant of time growing droplets with $R>R^{*}$ are still described by Eq. (A12), and, in particular, their contact angle has the equilibrium value $\theta_{c}$.

However, this description cannot apply when we have a substrate with a chemical inhomogeneity of extent $b$ (cf. Fig. 1). We assume here conditions (corresponding to our actual choice of the boundary fields $H_{w 1}, H_{w 2}$, and $H_{w 3}$ ) where nucleation rates in the region where wall fields $H_{w 1}, H_{w 2}$ act are negligibly small; so only nucleation within the region of the chemical inhomogeneity needs to be considered, i.e., circlecut shaped droplets with baseline $b_{\text {drop }}=2 R \sin \left(\theta_{c}\right)$ [Eq. (A1)] smaller than $b$. Such droplets can grow at constant contact angle with time only until $b_{d r o p}=b_{d r o p}^{*}=b$ and then either get pinned and grow in area and angle up to some nontrivial values, which we shall study in this section or they "depin" and grow with baseline $b_{d r o p}>b, b_{d r o p}=2 R \sin \left(\pi-\theta_{c}\right)$.

Thus we turn to an analysis of the regime where $b_{d r o p} \simeq b$. In this regime, we have to use Eq. (A9) for the area of the droplet and hence write the free energy of the droplet as

$$
\Delta F_{\text {drop }}=\text { constant }+f_{\text {int }} 2 R \theta-2 m_{\text {coex }} H_{b} R^{2}\left[\theta-\frac{1}{2} \sin (2 \theta)\right] \text {, }
$$

where the constant is fixed by the requirement that for $\theta=\theta_{c}$ and $b_{d r o p}=b$ the previous expression for $\Delta F_{d r o p}$ [Eq. (A12)] results, i.e., constant $=-b f_{\text {int }} \cos \left(\theta_{c}\right)$. Note that now $R$ is not $R^{*}$ but rather $R=b /(2 \sin (\theta))$ from geometry (see Fig. 20 in Appendix A). Thus we obtain, eliminating $R$ in favor of $b /(2 \sin (\theta))$,

$$
\begin{aligned}
\Delta F_{\text {drop }} / b f_{\text {int }}= & -\cos \left(\theta_{c}\right)+\frac{\theta}{\sin (\theta)} \\
& -\frac{m_{\text {coex }} H_{b} b}{2 f_{\text {int }}}\left[\frac{\theta}{\sin ^{2}(\theta)}-\frac{\cos (\theta)}{\sin (\theta)}\right] .
\end{aligned}
$$

Now the angle $\theta$ is found from the condition

$$
\frac{\partial}{\partial \theta}\left(\Delta F_{d r o p} / b f_{\text {int }}\right)=0,
$$

which after simple algebra yields the minimum of the free energy for

$$
\sin \left(\theta_{\text {min }}\right)=m_{\text {coex }} H_{b} b / f_{\text {int }}, \quad \theta_{c}<\theta_{\text {min }}<\pi / 2,
$$

while the angle $\theta_{\max }=\pi-\theta_{\min }$ is also a solution of Eq. (22) but corresponds to the maximum of the free energy. Note that the condition $\theta_{c}<\theta_{\text {min }}$ has been added since Eq. (21) makes sense only for $\theta>\theta_{c}$.

Using this result, it is straightforward to evaluate the area $A$ of the droplet and hence the excess density $\Delta \rho$ due to the droplet. Figure 12(a) presents plots of $\theta$ versus $\Delta \rho / b^{2}$, comparing Eq. (A9) to the simulation data in the regime $20^{\circ}<\theta<70^{\circ}$. The agreement is reasonable, in particular since no adjustable parameter whatsoever is present. Note that the knowledge of the contact angle $\theta_{c}$ is not needed here (apart from defining the range on which this relationship should be used).

Figure 12(b) presents a plot of $\theta$ versus $b H_{b}$. The dashed horizontal line shows the estimate of $\theta_{c}$ from the SOS approximation Eq. (19). The actual variation of $\theta$ with $b H_{b}$ [Fig. 7(b)] should be compared to this figure only for $b H_{b}>1$ since for small fields, where the excess mass due to the droplet is small, the assumptions of the above quasi-macroscopic analysis clearly are inapplicable. At least, for $b H_{b} \geq 1.5$ the prediction is close to the observations from the simulations [Fig. 7(b)]; furthermore, the values of $\theta_{\text {eff }}^{\max }$ obtained for
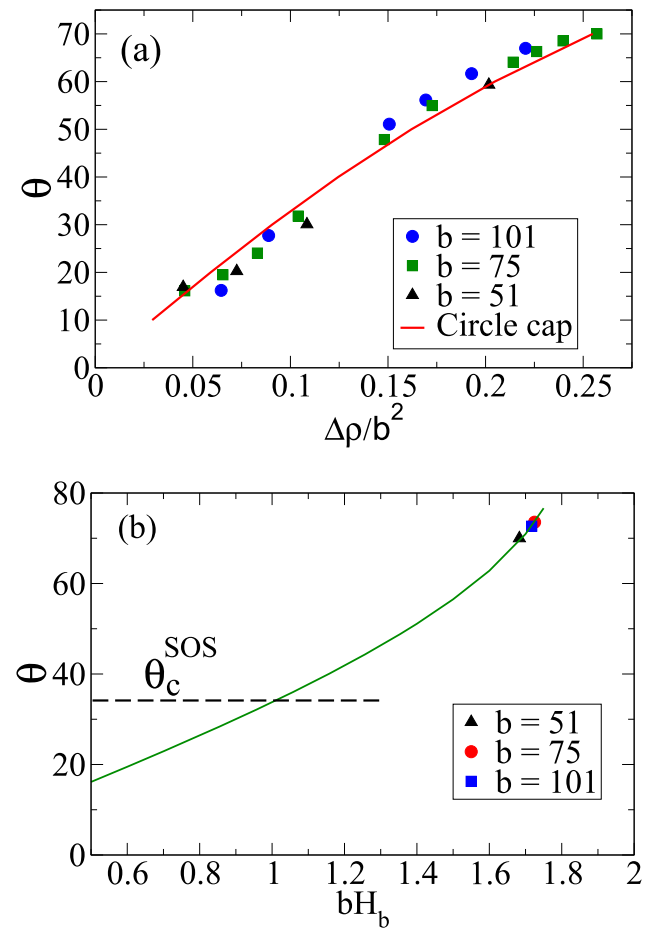

FIG. 12. (a) Plots of $\theta$ versus $\Delta \rho / b^{2}$, comparing Eq. (A9) to the simulation data, obtained for $t=0.40$, in the regime $20^{\circ}<\theta<70^{\circ}$. (b) The full line shows a plot of $\theta$ versus $b H_{b}$, according to Eq. (23) taken $f_{\text {int }}=1.7987$ for $t=0.40 .{ }^{48}$ The dashed horizontal line shows the estimate of $\theta_{c}$ from the SOS approximation Eq. (19). Also, simulation results of $\theta_{e f f}^{\max }$ obtained for different values of $b$ are shown by means of symbols. 


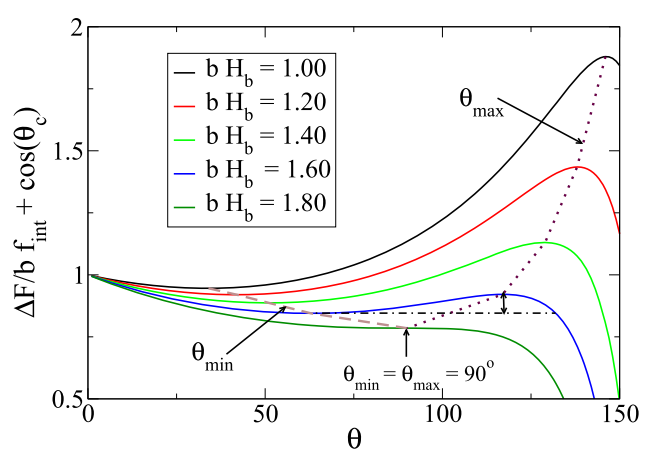

FIG. 13. Plots of the free energy $\Delta F / b f_{\text {int }}+\cos \left(\theta_{c}\right)$ given by Eq. (21) as a function of $\theta$ for various values of the parameters $b H_{b}$, as indicated. Plots obtained for $\theta_{c}=34^{\circ}$ and $f_{\text {int }}=1.7987$, which correspond to $t=0.4$. The dashed (dotted) line shows the location of the minimum (maximum) of Eq. (21), as given by Eq. (23). Note that both lines merge at $\theta=\pi / 2$ such that $\theta_{\min }=\theta_{\max }$. The double arrow shows the free energy barrier $\Delta F_{2} / b f_{\text {int }}$ given by Eq. (26), for the choice $b H_{b}=1.6$.

the larger inhomogeneities, i.e., $51 \leq b \leq 101$, are in full agreement with the theoretical result given by Eq. (23) taken $f_{\text {int }}=1.7987$ for $t=0.40^{48}$ ( $f_{\text {int }}$ is taken in units of $J$ ).

It really is illuminating to plot the free energy given by Eq. (21) as a function of $\theta$ for various values of $b H_{b}$, as shown in Fig. 13. In fact, both the minimum and the maximum of the free energy can clearly be observed. Furthermore, the free energy difference between the maximum and the minimum $\left(\Delta F_{2} / b f_{\text {int }}\right)$ monotonically decreases when $b H_{b}$ increases and finally vanish where $\theta_{\min }$ and $\theta_{\max }$ merge at $\theta_{\min }=\theta_{\max }=\frac{\pi}{2}$. A more quantitative evaluation of the free energy barrier can be performed by reinserting Eq. (23) in the free energy $\Delta F_{\text {drop }}$ [Eq. (21)], obtaining the following relationships for the minimum

$$
\Delta F_{d r o p}^{\min } / b f_{\text {int }}=-\cos \left(\theta_{c}\right)+\frac{1}{2}\left[\frac{\theta_{\text {min }}}{\sin \left(\theta_{\min }\right)}+\cos \left(\theta_{\min }\right)\right]
$$

and the maximum

$$
\Delta F_{\text {drop }}^{\max } / b f_{\text {int }}=-\cos \left(\theta_{c}\right)+\frac{1}{2}\left[\frac{\pi-\theta_{\min }}{\sin \left(\theta_{\min }\right)}-\cos \left(\theta_{\min }\right)\right] .
$$

Then, the difference between the maximum and the minimum of the free energy is given by

$$
\Delta F_{2} / b f_{\text {int }}=\frac{\left(\frac{\pi}{2}-\theta_{\min }\right)}{\sin \left(\theta_{\min }\right)}-\cos \left(\theta_{\min }\right)
$$

This difference provides the height of the barrier which prevents that the system can move from the angle $\theta_{c}$ to the angle $\pi-\theta_{c}$. Such a move is needed for the droplet to subsequently grow increasing its baseline beyond $b$ at fixed angle $\pi-\theta_{c}$. Expanding Eq. (26) in terms of the angle $\pi / 2-\theta_{\min }=\alpha$, one gets $\Delta F_{2} / b f_{\text {int }} \simeq \frac{2}{3}\left(\alpha^{3}\right)$. For $\theta_{\min }=70^{\circ}\left(\alpha=20^{\circ}\right)$, this leads to a barrier (in units of $k_{B} T$ ) of about 2.6.

Actually, when $m_{\text {coex }} b H_{b}$ approaches $f_{\text {int }}$, then the angles where the minimum and maximum of the free energy occur merge at $\theta=90^{\circ}$ (cf. Fig. 13 for $b H_{b}=1.8$ ). However, already at a smaller field (keeping $b$ constant) the barrier caused by the free energy maximum, $\Delta F_{2}$ given by Eq. (26), will be small enough so that the second nucleation event by which the angle grows from $\theta_{c}$ to $\pi-\theta_{c}$ can take place. Note that the analytical formula for the barrier can also be extracted from Eqs. (21) and (23), and Fig. 13 shows that long before $\theta$ reaches $90^{\circ}$ it will
TABLE I. List of the bulk fields ( $H_{b}^{\max }$, 3rd column) where the jump of the excess density indicating the formation of the liquid phase is observed, as measured for several choices of the temperature $t$ (1st column), given in units of the bulk critical temperature of the Ising model. Also, the interface tension $f_{\text {int }}$ given by the Onsager exact solution ${ }^{48}$ and the apparent angle $\theta_{\min }$, as determined by using Eq. (23), are listed in the second and fourth columns, respectively. Data obtained by taking $b=51$ for the length of the inhomogeneity. Note that these transition fields can be estimated only with a relative error of about one percent, and a similar error is expected for $\theta_{\min }$. Both $f_{\text {int }}$ and $H_{b}^{\max }$ are given in units of $J$.

\begin{tabular}{lccc}
\hline \hline$t$ & $f_{\text {int }}$ & $H_{b}^{\max }$ & $\theta_{\min }(\mathrm{deg})$ Eq. (23) \\
\hline 0.30 & 1.92780 & $0.0354(2)$ & 69.5 \\
0.325 & 1.90190 & $0.0350(2)$ & 69.8 \\
0.350 & 1.87168 & $0.0339(2)$ & 67.5 \\
0.375 & 1.80873 & $0.0334(2)$ & 70.4 \\
0.3875 & 1.81848 & $0.0326(2)$ & 66.1 \\
0.4000 & 1.79873 & $0.0330(2)$ & 70.0 \\
0.425 & 1.75625 & $0.0315(2)$ & 66.2 \\
0.430 & 1.74728 & $0.0308(2)$ & 64.0 \\
0.440 & 1.72851 & $0.0304(2)$ & 63.8 \\
0.445 & 1.71954 & $0.0302(2)$ & 63.6 \\
\hline \hline
\end{tabular}

be of order of a few $k_{B} T$ only. This argument also explains why the temperature dependence of the apparent angle (close to $70^{\circ}$ ) where the depinning transition occurs is rather weak (see Table I): the scale for the barrier is simply set by $b f_{\text {int }}$, and this quantity does not vary strongly with $T$ for the choices we have made. Thus when this barrier is small enough, the instability that would occur for $\theta=90^{\circ}$ (where $\theta_{\text {min }}$ and $\theta_{\max }$ merge) is pre-empted by the jump of the angle $\theta$ from $\theta_{c}$ to $\pi-\theta_{c}$.

On the other hand, one can change the height of the barrier by around one order of magnitude just by taking a fixed temperature $(t=0.4)$, such as $f_{\text {int }}=1.79873$, but varying the length of the inhomogeneity $13 \leq b \leq 101$. In this way, an increment of the apparent angle of about $20^{\circ}$ is observed, as shown in Table II. It is also obvious from Eq. (23) that solutions for $\theta_{\min }$, corresponding to pinned droplets, exist only for

TABLE II. List of the apparent angles $\theta_{\min }$ (fourth column), as determined by using Eq. (23) for different choices of the length of the inhomogeneity $b$ (first column). Notice that the bulk fields ( $H_{b}^{\max }, 3$ rd column) where the jump of the excess density indicating the formation of the liquid phase is observed depend on $b$. Data taken at $t=0.40$ so that the interface tension is given by $f_{\text {int }}$ $=1.79873$ according to the Onsager exact solution ${ }^{48}$ however, the value of $b f_{\text {int }}$ (second column) that sets the height of the free energy barrier changes almost one order of magnitude for the choices of $b$ that are used.

\begin{tabular}{lccc}
\hline \hline$b$ & $b f_{\text {int }}$ & $H_{b}^{\max }$ & $\theta_{\min }(\mathrm{deg})$ Eq. (23) \\
\hline 13 & 23.38346 & $0.110(5)$ & 52.7 \\
17 & 30.57838 & $0.088(3)$ & 55.8 \\
21 & 37.77329 & $0.075(3)$ & 61.1 \\
25 & 44.96820 & $0.062(2)$ & 59.5 \\
31 & 55.76057 & $0.051(2)$ & 61.5 \\
39 & 70.15039 & $0.040(2)$ & 60.1 \\
45 & 80.94276 & $0.036(2)$ & 64.2 \\
51 & 91.73513 & $0.033(2)$ & 70.0 \\
75 & 134.90475 & $0.023(2)$ & 73.5 \\
101 & 181.67173 & $0.017(2)$ & 72.7 \\
\hline \hline
\end{tabular}


$m_{\text {coex }} H_{b} b / f_{\text {int }}<1$ : for larger fields, droplet growth with time is not hindered by any barriers, after they have been nucleated.

The smooth variation of $\theta$ from $\theta_{c}$ to $\pi-\theta_{c}$ with increasing droplet area as predicted by Lipowsky et al. ${ }^{65-67}$ is a special consequence of the canonical ensemble, where the droplet volume (in $d=3$ ) or the droplet area (in $d=2$ ) is taken as a fixed independent variable. By contrast, only part of the variation is realizable as a metastability effect in the grand canonical ensemble, where $H_{b}$ is given. According to the theory outlined above, metastable pinned droplets should exist only up to a "spinodal" where $\theta_{\min }=\frac{\pi}{2}$. The spinodal field is then $H_{b}^{\text {spin }}=f_{\text {int }} / b m_{\text {coex }}$ such that $H_{b}^{\text {spin }} / H_{b}^{\text {crit }}=1 / \sin \left(\theta_{c}\right)$ [see also Eqs. (18) and (23)]. However, it should be kept in mind that for systems with short-range interactions "spinodals" are a somewhat ill-defined concept ${ }^{2}$ and cannot be reached in practice. In the context of nucleation phenomena, the present case of a grand canonical ensemble is the physically meaningful choice, of course.

For understanding of the results observed in the simulations, it is hence crucial to consider the combined effects of the primary nucleation event of the wall-attached droplet and a further growth of this droplet.

We have made the hypothesis that for this growth a "local equilibrium" assumption holds, in particular near the point where the droplet-vapor interface meets the substrate. This implies, for the case where the length $b_{d r o p}$ of the growing droplet is still less than $b$, that we have $\theta=\theta_{c}$ for the contact line of the growing droplet (see Fig. 20 in Appendix A, top panel). However, for droplets that have $b_{\text {drop }}>b$, we have $\theta=\pi-\theta_{c}$ (see Fig. 20 in Appendix A, lower panel). For fields $H_{b}<H_{b}^{\text {spin }}=f_{\text {int }} /\left(b m_{\text {coex }}\right)$, metastable pinned droplets are predicted, and a barrier $\Delta F_{2}$ for the "depinning" of these droplets could be estimated [Eq. (26)]. The actual limit of stability of metastable pinned droplets, as seen in Figs. 7 and 8, is somewhat smaller than $H_{b}^{\text {spin }}$ : this happens because when the barrier $\Delta F_{2}$ is small, it can be overcome in a second nucleation event.

Of course, a perfect quantitative agreement of the predictions based on our phenomenological theory for pinned droplets with the corresponding simulation results should not be expected: (i) the mean-field like treatment of Eqs. (19)(23) disregards the huge statistical fluctuations that are present (Fig. 2), (ii) the anisotropy of the interfacial free energy should lead to some deviations of the shape of the pinned droplets from the circle cut, which should cause some systematic deviations from the free energy plotted in Fig. 13. Also, the curvature of the interface may modify the effective surface tension.

\section{NUCLEATION KINETICS AND DROPLET GROWTH}

Already in earlier work on studies of homogeneous nucleation in bulk Ising models (see, e.g., Ref. 33 for a recent review), it has been shown that one needs to distinguish two very different time scales for conditions where the phase transformation is caused by nucleation and growth of a single droplet. One timescale is the typical lifetime of the metastable state, which is then simply inversely proportional to the nucleation rate; and the second timescale is the time needed for the nucleated droplet to grow and essentially occupy the total volume of the simulation box. However, often these processes are somewhat confused by the crossover to the regime where during the phase transformation many droplets are nucleated in different parts of the system and the lifetime of the metastable state is then limited by this competitive growth of many droplets. This latter regime is dominant when the simulation volume is relatively large and $H_{b}$ is not so small, so nucleation becomes relatively easy. ${ }^{2}$

In the present work, conditions were chosen such that homogeneous nucleation is not observable at all, and heterogeneous nucleation is restricted to the boundary region of length $b$. It is then rather straightforward to follow the growth of the single droplet (Fig. 11), and it turns out that the time intervals between the snapshots of the growing droplet are indeed very small in comparison with the nucleation times. In order to give further insight on the involved times as well as on the growing and nucleation process of the droplets, Fig. 14 shows plots of the time evolution of both the total excess density $\Delta \rho$ due to the droplet (upper panel) and the linear density excess $\Delta \rho_{\perp}$ measured in the direction perpendicular to the wall just at the center of the droplet. Each curve is the average over several hundred individual time evolutions of the system. In Fig. 14(a), one can roughly estimate the average time required by the system to achieve the metastable state $\left(\tau_{M S}\right)$, which for the case shown (i.e., $t=0.40$ and $b=51$ ) is $\tau_{M S} \simeq 10^{4}$ MCS. Choosing $H_{b}=0.030$, no nucleation events are detected during the observation time $\left(\tau_{o b s}=10^{6}\right)$, and the curve remains flat after achieving the metastable state. For $H_{b}=0.032$, few nucleation events are detected, and each of them shows up as an upward step in the corresponding plot. The height of each
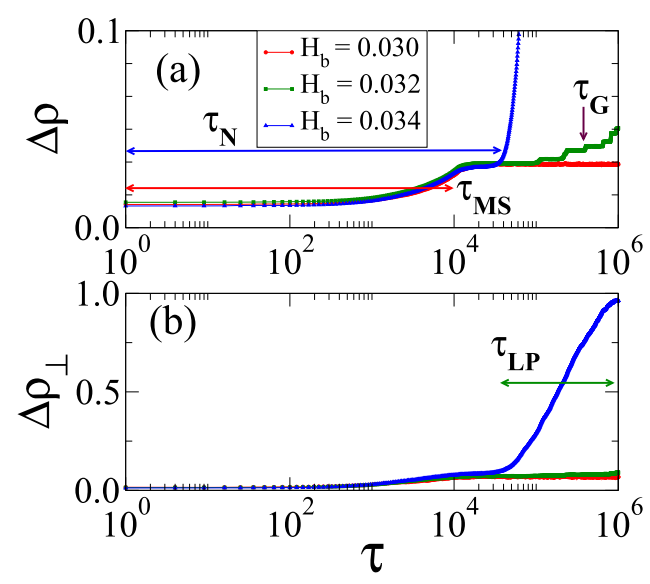

FIG. 14. (a) Linear-log plot of the density versus time $(\tau)$ as obtained for $t=0.40, b=51$, and different choices of the bulk field $H_{b}$, as indicated. The horizontal lower and upper double arrows indicate the average times required to achieve the metastable state and the nucleation time, $\tau_{M S} \simeq 10^{4} \mathrm{MCS}$ and $\tau_{N} \simeq 4 \times 10^{4} \mathrm{MCS}$, respectively. Also, the vertical arrow shows a single growth event of an already nucleated droplet with a characteristic growth time given by $\tau_{G} \simeq 10^{4} \mathrm{MCS}$. Note that the fields $H_{b}$ included here are all slightly less than the predicted "spinodal" (ultimate stability limit) $H_{b}^{\text {spin }} \simeq 0.0353$ : hence here droplet growth involves the second nucleation event, in which the (small) barrier $\Delta F_{2}$ is overcome [see Eq. (26) and Fig. 13]. (b) Linear$\log$ plot of the linear density as measured at the center of the sample and in the direction perpendicular to the wall $\left(\Delta \rho_{\perp}\right)$ versus time as obtained for the same parameters as in (a). The horizontal double arrow shows the average time needed to cover the whole sample for $H_{b}=0.034$ with the liquid phase, $\tau_{L P} \simeq 8 \times 10^{5} \mathrm{MCS}$. Averages were taken over 386, 453, and 318 different realizations for $H_{b}=0.030, H_{b}=0.032$, and $H_{b}=0.034$, respectively. More details in the text. 
individual step simply is the inverse of the number of runs since in each run when nucleation has occurred the droplet grows fast (on the scale of $\tau_{N}$ ) to fill the available area. Here, one can estimate the typical growth time $\left(\tau_{G}\right)$ required for each already nucleated droplet to expand over the whole sample, namely, $\tau_{G} \simeq 10^{4}$ MCS. However, for $H_{b}=0.034$ nucleation is dominant and one can estimate $\tau_{N} \simeq 4 \times 10^{4}$ (also by discounting $\tau_{M S}$ one can get $\tau_{N} \simeq 3 \times 10^{4} \mathrm{MCS}$ ). On the other hand, the time evolution of the density per unit length as measured in the direction perpendicular to the sample [Fig. 14(b)], which shows the development of the droplet in that direction, is fully consistent with the above discussed scenario. Furthermore, here one can also estimate the average time elapsed between the onset of nucleation and the achievement of a full liquid phase covering the whole sample, $\tau_{L P} \simeq 8 \times 10^{5} \mathrm{MCS}$. Note that this averaged time results from the contribution of many growing events of already nucleated droplets, occurring at different times over a wide time interval (actually 318 events for $H_{b}=0.034$ ), each of them having a short lifetime of the order of $\tau_{G} \simeq 10^{4} \mathrm{MCS}$, as already discussed.

The individual nucleation events seen in Fig. 14 actually all relate to overcoming the barrier $\Delta F_{2}$ discussed in Fig. 13 since the fields $H_{b}$ all are slightly below the stability limit $H_{b}^{\text {spin }}$.

For a more quantitative analysis, we have also recorded both the nucleation time distribution $\left(P\left(\tau_{N}\right)\right)$ and the growth time distribution $\left(P\left(\tau_{G}\right)\right)$ for the case $t=0.40, b=51$, and for different choices of $H_{b}$, as shown in Figs. 15(a) and 15(b), respectively.

These choices all refer to $H_{b}>H_{b}^{\text {spin }}$, and hence, for them the barrier $\Delta F_{2}$ does no longer occur. A simple comparison of both figures indicates that the characteristic times, as estimated from the location of the peaks of the distributions, are roughly of the same order for larger fields $\left(H_{b} \geq 0.08\right)$, while $\tau_{N}>\tau_{G}$ in the opposite limit. In fact, Fig. 16(a) shows the monotonic increase of the ratio $\tau_{G} / \tau_{N}$ when it is plotted versus $H_{b}$, spanning the range $0.25 \leq \tau_{G} / \tau_{N} \leq 1$. Also, Fig. 16(b) shows a plot of $\tau_{N}$ versus $1 / H_{b}$. Nucleation theory predicts

$$
\ln \left(\tau_{N}\right) \propto \frac{\Delta F^{*}}{k_{B} T}=\frac{\pi}{2} f_{\text {int }}^{2} \frac{1}{\left(\rho_{\ell}^{\text {coex }}-\rho_{v}^{\text {coex }}\right) H_{b}} \frac{f_{V T}}{k_{B} T} .
$$

However, the curvature of the log-linear plot indicates that only part of the chosen region of fields is in the regime where the
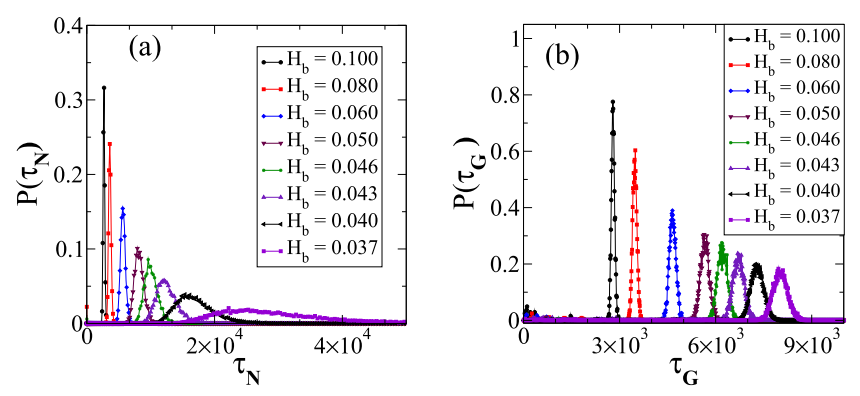

FIG. 15. (a) Plot of the nucleation time distribution function $P\left(\tau_{N}\right)$ versus time as obtained for different values of the bulk field $H_{b}$ as indicated. Results correspond to $t=0.40, b=51$. Data averaged over $5 \times 10^{3}$ and $15 \times 10^{3}$ different initial configurations for $H_{b} \geq 0.043$ and $H_{b}<0.040$, respectively. (b) Plot of the growth time distribution function $P\left(\tau_{G}\right)$ versus time as obtained for the same choice of parameters as in (a).
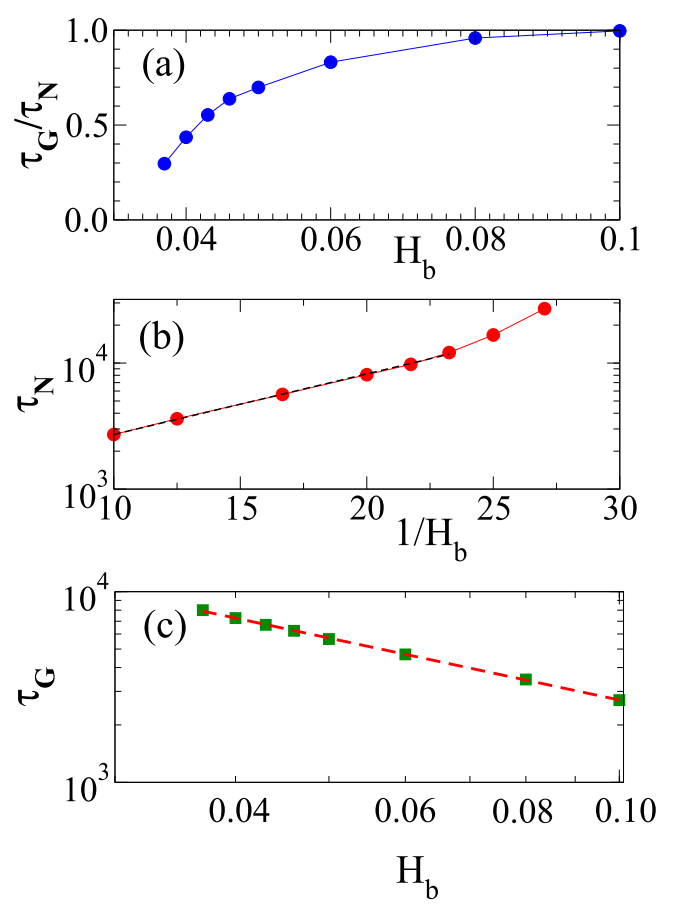

FIG. 16. (a) Plots of the ratio $\tau_{G} / \tau_{N}$ versus $H_{b}$ for the case $b=51$. The characteristic times are obtained from estimations of the peaks of the distributions shown in Fig. 15. (b) Log-linear plot of $\tau_{N}$ versus $1 / H_{b}$. The fit of the data within the linear regime $\left(H_{b}>0.04\right)$ shown by a dashed line yields $\Delta F^{*} H_{b} / k_{B} T=0.111$. (c) Plot of $\tau_{G}$ versus $H_{b}$ on logarithmic scales. The straight line corresponds to the best fit of the data with slope $-1.08(8)$ in agreement with the expected theoretical dependence, namely, $\tau_{G} \propto H_{b}^{-1}$. More details in the text.

barrier due to the heterogeneous nucleation on the inhomogeneity controls the kinetic exclusively; in fact, when $H_{b}$ approaches $H_{b}^{\text {spin }}$, a slowing down related to the barrier $\Delta F_{2}$ that occurs for $H_{b}<H_{b}^{\text {spin }}$ may be present. The best fit of the data of Fig. 16(b), within the linear regime, yields $\frac{\Delta F^{*} H_{b}}{k_{B} T}=0.111$. This number is smaller than the theoretical expectation given by $\frac{\pi}{2} f_{\text {int }}^{2} \frac{1}{\left(\rho_{\ell}^{\text {coex }}-\rho_{v}^{\text {coex }}\right)} \frac{f_{V T}}{k_{B} T}$ [see Eq. (27)], obtained by taking $f_{\text {int }}=1.7987,{ }^{48}$ which yields $\frac{\Delta F^{*} H_{b}}{k_{B} T}=0.231$ for $\theta_{c}=34^{\circ}$ in the Volmer-Turnbull factor [Eq. (A15)]. It is a subtle issue to understand where this discrepancy of about a factor of two in the effective barrier height comes from. One immediate thought concerns the curvature dependence of the interfacial free energy $f_{\text {int }}(R)$. In $d=2$, there is evidence from field theoretical calculations, ${ }^{68}$ Monte Carlo simulations of cluster-size distributions, ${ }^{69}$ and analysis of the two-phase coexistence $^{70}$ that

$$
\frac{f_{\text {int }}(R)}{f_{\text {int }}(\infty)}=1+\frac{5}{4 \pi f_{\text {int }}(\infty)} \frac{\ln (R)}{R}+\frac{\text { const }}{R},
$$

where the constant in the last term on the right-hand side is non-universal, while the prefactor $\frac{5}{4 \pi}$ of the logarithmic term is universal. If only this correction would be taken into account, the interfacial tension be enhanced by a factor $1+0.221 \frac{\ln (R)}{R}$, which for typical values of $R$ (e.g., $R=16$ ) is an enhancement of about $4 \%$. Neither the magnitude nor the sign of this effect can account for the observed discrepancy. Actually a more plausible assumption is that our estimate of the 

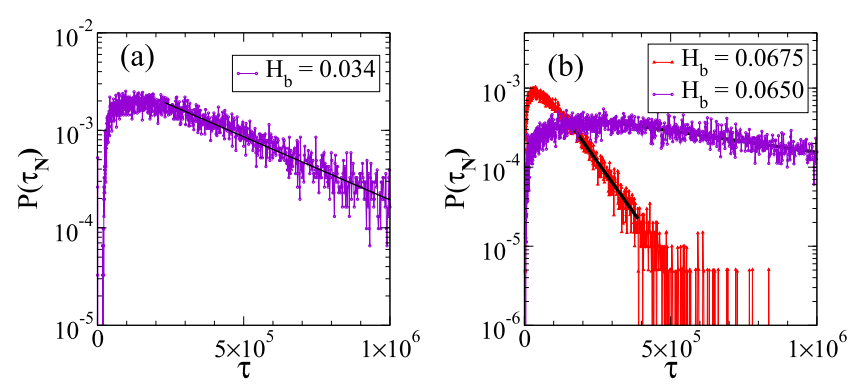

FIG. 17. Log-linear plots of $P\left(\tau_{N}\right)$ versus time as obtained for $t=0.40$ and different choices of the bulk field as indicated. (a) Results corresponding to $b=51$. After a waiting time $\left(\tau_{w} \simeq 2 \times 10^{4} \mathrm{MCS}\right.$ in this case), the distribution exhibits an exponential decay with a characteristic nucleation time $\tau_{N} \simeq 3.36$ $\times 10^{5} \mathrm{MCS}$, as obtained from the slope of the fitted curve as shown by means of a full line. (b) Results corresponding to $b=25$. By following the procedure described in (a), the fitted characteristic nucleation times are $\tau_{N} \simeq 7.78$ $\times 10^{5} \mathrm{MCS}$, and $\tau_{N} \simeq 8.40 \times 10^{4} \mathrm{MCS}$, for $H_{b}=0.0650$ and $H_{b}=0.0675$, respectively. In both cases, the slopes of the fitted curves are shown by means of full lines. Note that in both cases the fields are slightly less than $H_{b}^{\text {spin }}=0.072$, and hence the combined effect of heterogeneous nucleation on the wall inhomogeneity and depinning of the metastable droplet matters.

contact angle $\theta_{c}$ and hence the factor $f_{V T}\left(\theta_{c}\right)$ is an overestimate. Since $f_{V T}\left(\theta_{c}\right) \approx \frac{4}{3 \pi} \theta^{3}$, a decrease of $\theta_{c}$ by a few degrees already suffices to reduce $f_{V T}\left(\theta_{c}\right)$ by a factor of two, e.g., $f_{V T}\left(27^{\circ}\right) / f_{V T}\left(34^{\circ}\right) \simeq 0.51$. Moreover the equation for $f_{V T}\left(\theta_{c}\right)$ holds only for circle-cut shaped droplets, and the effect of anisotropy causing somewhat non-circular shapes (see Fig. 19 in Appendix A) of the droplet on $f_{V T}\left(\theta_{c}\right)$ still needs to be clarified. Also, the curvature of the plot shown in Fig. 16(b) may be taken as an indication that it is questionable whether the asymptotic region where the theory holds has been reached. Thus clearly the conclusion emerges that in spite of the simplicity of the Ising model still more work is needed to understand there heterogeneous nucleation quantitatively.

Figure 16(c) shows a log-log plot of $\tau_{G}$ versus $H_{b}$ to show that the growth time $\tau_{G}$ scales inversely with $H_{b}$, as expected. On the other hand, Fig. 17 shows log-linear plots of $P\left(\tau_{N}\right)$ versus $\tau_{N}$, for different choices of the length of the inhomogeneity $b$ and the bulk field $H_{b}$, demonstrating an exponential distribution for the long times, as theoretically expected. ${ }^{56,57}$

Of course, it would be interesting to explore the kinetics of heterogeneous nucleation systematically for a wide range of $b$, but due to excessive needs for computer time this has not been attempted. So, we have only studied one other choice, $b=25$. For $b=25$, one has $H_{b}^{\text {spin }} / J \simeq 0.060$, so the measurements performed slightly above $H_{b}^{\text {spin }}$ indicate that $\tau_{N}$ depends strongly on $H_{b}$, and decrease for larger values of $H_{b}$, as expected. The fact that $\tau_{N}$ for $b=51$ and $H_{b} / J=0.034$ is smaller than for the case $b=25$ and $H_{b} / J=0.064$ means that one has smaller nucleation times for larger inhomogeneities, since the larger the inhomogeneity is, the less tendency is found that the nucleated droplet gets pinned.

\section{CONCLUSIONS}

In this work, we have considered the effect of a chemical inhomogeneity on heterogeneous nucleation on a flat substrate. As a generic case, we have considered the simple Ising lattice gas system in $d=2$ dimensions, where the flat substrate is just a straight line, and the chemical inhomogeneity is represented by a positive boundary field $H_{w 3}$ (favoring the liquid phase of the lattice gas) acting on a part of this line of length $b$, while in the remaining part of this lower boundary of the system a boundary field $H_{w 2}=-H_{w 3}$ is applied favoring the vapor phase of the lattice gas. For the Monte Carlo simulations of our model, we choose a boundary with finite length $M$ and periodic boundary condition in the direction parallel to this boundary, while in the direction perpendicular to this boundary a finite linear dimension $L$ is used, and at the upper boundary, a negative field $H_{w 1}=H_{w 2} / 4$ acts, to stabilize the vapor as a bulk phase of the system in the absence of a bulk field, $H_{b}=0$. For conditions of partial wetting, the density inhomogeneity in the lattice gas caused by the chemical inhomogeneity then extends only over a distance of the order of one lattice spacing, for $H_{b}=0$ [Fig. 3(a)]. However, when a small field $H_{b}>0$ is applied, the vapor phase chosen as the initial state of the system becomes metastable, and the structure of the density inhomogeneity caused by the chemical inhomogeneity, Figs. 3-9, as well as the decay rate of the metastable state due to nucleation of a boundary-attached droplet and its growth, Figs. 10-16, are the subjects of investigation. Conditions are chosen such that neither homogeneous nucleation in the bulk nor nucleation starting in the boundary regions favoring the vapor phase can ever be observed.

For each choice of temperature, and the width $b$ of the chemical inhomogeneity, as well as for many fields $H_{b}$, we perform many hundred equivalent Monte Carlo runs, differing by the pseudorandom numbers used to realize the time evolution of the Monte Carlo sampling process. By using over $20 \times$ $10^{6}$ Monte Carlo steps per lattice site (MCS) per site, we are able to reliably estimate various relaxations times and their distributions (Figs. 15-17) over 6 decades of time. The initial stages of the relaxation process are characterized by the equilibration of the metastable state, after the field $H_{b}$ has been switched on at time $\tau=0$, taking a time $\tau_{M S}$. If $H_{b}$ is small enough, e.g., $H_{b} \leq 0.030$ for the choice $t=0.40, b=51$, no decay of the metastable state is observed, which implies that the nucleation time $\tau_{N}$ exceeds the observation time $\tau_{o b s}$.

If we would study heterogeneous nucleation on a chemically homogeneous boundary of linear dimension $M$, the nucleation time (for the regime of fields where single-droplet nucleation matters) would be related to the nucleation rate $J^{\text {het }}$ by $\tau_{N}=\left(M J^{h e t}\right)^{-1}$. When a nucleation event has occurred, it takes a time $\tau_{G}$ for the critical droplet to grow until the whole (finite) system has transformed; only when $\tau_{N} \gg \tau_{G}$ is the phase transformation triggered by single nuclei, the dominant process. In the regime where $\tau_{N}$ and $\tau_{G}$ are comparable, the simultaneous growth of multiple nucleated droplets needs to be considered, making separate estimations of $\tau_{N}$ and $\tau_{G}$ difficult. By choosing our geometry with a chemical inhomogeneity, we extend the regime where the transformation triggered by single nuclei is the dominant process: note that the baseline of the critical droplet is $b_{d r o p}^{*}=2 R^{*} \sin \left(\theta_{c}\right)$, where $R^{*}$ is the critical droplet radius and $\theta_{c}$ is the contact angle, assuming droplets of circle-cut shape; only when $b_{\text {drop }} \ll b$, phase transformations affected by nucleation of multiple droplets and their competitive growth could matter. The detailed analysis of our observed 
phase transformation events has allowed us a separate analysis of the distributions of $\tau_{N}$ and $\tau_{G}$; as theoretically expected, the growth rate of supercritical droplets is proportional to $H_{b}$, and hence $\tau_{G} \propto 1 / H_{b}$ [Fig. 16(c)], while $\tau_{N}$ varies exponentially with $1 / H_{b}, \ln \tau_{N} \propto 1 / H_{b}$ [Fig. 16(b)], as expected from nucleation theory in $d=2$ dimensions. Unfortunately, only a very small range of $H_{b}$, much less than a decade [Fig. 16(b)], is available when the time scales for nucleation and growth are well separated.

Very interesting behavior was found for the metastable regime, where during observation time $\tau_{o b s}$ no phase transformation occurs. On general grounds, one can predict that then the chemical inhomogeneity causes an excess density $\Delta \rho$ in the system, which exhibits a scaling behavior $\Delta \rho=b^{2} \tilde{M}\left(t, b H_{b}\right)$, Eq. (17), the effective droplet height $h_{e f f} / b$ similarly is a function of the product $b H_{b}$ only [Fig. 3(c)], as well as the effective contact angle $\theta_{\text {eff }}$ [Fig. 7(b)]. We hence identified two regimes: for very small values of $H_{b}$ such that $b_{\text {drop }}>b$, nucleation of droplets with the contact angle $\theta_{c}$ "preferred" by the chemical inhomogeneity is still geometrically impossible; it does not matter how large observation times are chosen. In fact, in this regime critical droplets would have the shape as shown in Fig. 20 (lower part), their baseline $b_{\text {drop }}$ extending beyond $b$ and the contact angle being $\pi-\theta_{c}$, but the corresponding nucleation barriers correspond to astronomically large nucleation times and hence are of no interest here. In this regime, thermal fluctuations allow only the occasional formation of subcritical nuclei with $R<R^{*}$, of circle cut shape with contact angle $\theta_{c}$. Thus the average effect of such fluctuations is measured by the scaling function $\tilde{M}\left(t, b H_{b}\right)$ for $H_{b}<H_{b}^{c r i t}$, where $H_{b}^{\text {crit }}$ can be estimated as $H_{b}^{\text {crit }}=\sin \left(\theta_{c}\right) f_{\text {int }} /\left(b m_{\text {coex }}\right)$, when we ignore anisotropy effects on the interfacial tension in the lattice gas model. In any cases, $H_{b}^{\text {crit }}$ is the smallest field where a droplet with the correct contact angle $\theta_{c}$ fits to the chemical inhomogeneity. For $H_{b}^{\text {crit }}<H_{b}<H_{b}^{\text {spin }}$, we may encounter pinned droplets, having a baseline of
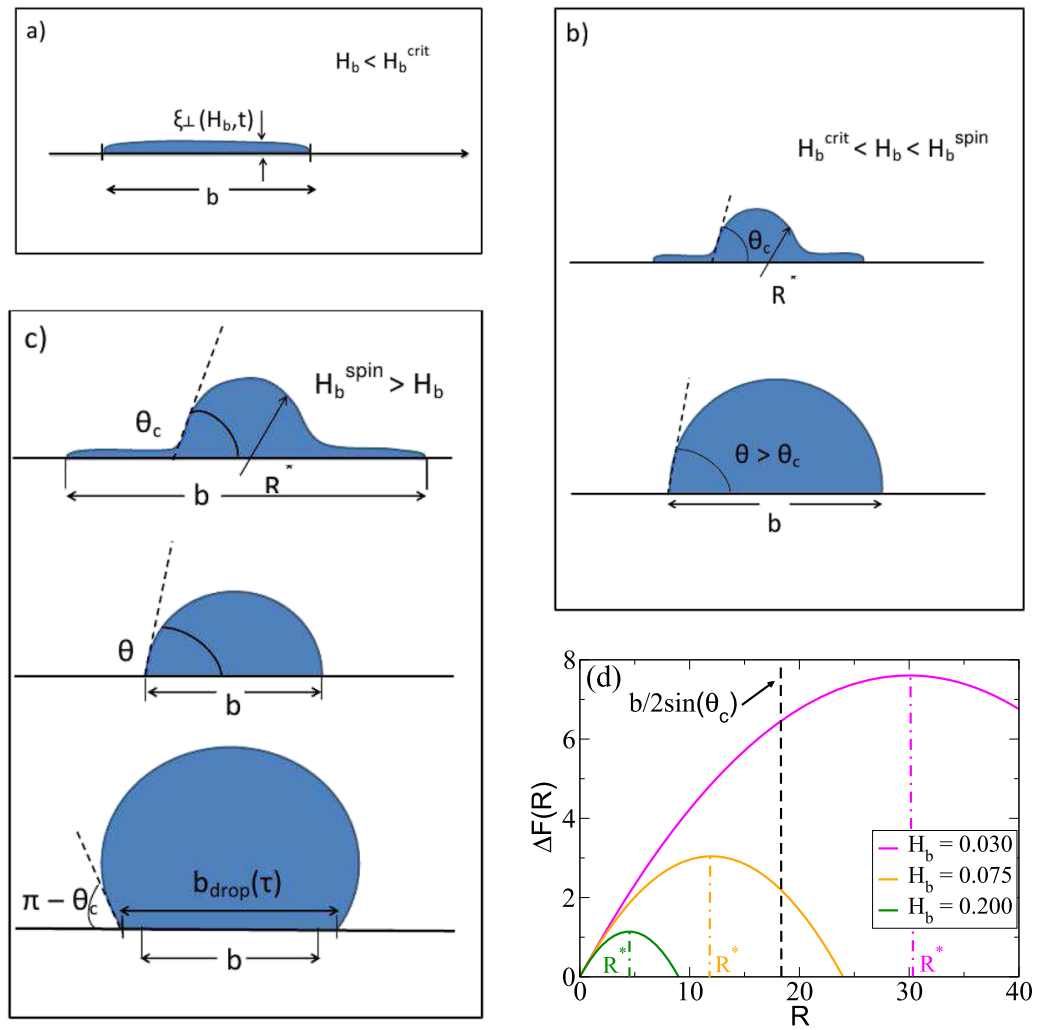

FIG. 18. Sketches summarizing qualitatively the different regions of wall-attached droplets for the case of an inhomogeneity of linear extension $b$ identified in this work. Case (a) shows the range where the bulk field $H_{b}$ is very small, namely, $H_{b}<H_{b}^{\text {crit }}\left(H_{b}^{\text {crit }}=f_{\text {int }} \sin \left(\theta_{c}\right) /\left(b m_{\text {coex }}\right)\right)$. Then the critical droplet having a contact angle $\theta_{c}$ would require a baseline larger than $b$, since then $R^{*}$ exceeds $b /\left(2 \sin \left(\theta_{c}\right)\right)$, see the case $H_{b}=0.030$ in part (d). No nucleation is then possible, and due to the average effect of subcritical droplets, a density excess $\Delta \rho \propto b \xi_{\perp}$ occurs on the inhomogeneity. Case (b) shows the regime $H_{b}^{c r i t}<H_{b}<H_{b}^{\text {spin }}$ $\left(H_{b}^{\text {spin }}=f_{\text {int }} /\left(b_{\text {coex }}\right)\right)$, where critical droplets of radius $R^{*}$ and contact angle $\theta_{c}$ with baseline $b_{d r o p}^{*}<b$ are nucleated, and their radius grows until their baseline is equal to $b$. Then these droplets can lower their free energy further by increasing their contact angle from $\theta_{c}$ to $\theta_{\text {min }}$. These metastable pinned droplets are characterized by $\sin \left(\theta_{\text {min }}\right)=m_{\text {coex }} H_{b} b / f_{\text {int }}$. The case $H_{b}=0.075$ in part (d) illustrates the corresponding $\Delta F(R)$ in the regime where $\theta=\theta_{c}$. Case (c) shows the behavior for $H_{b}^{\text {spin }}<H_{b}$, e.g., the case $H_{b}=0.20$ in part (d), where the critical droplet nucleates with contact angle, $\theta_{c}$, has such a small radius $R^{*}$ and corresponding baseline, that after growth to the baseline $b$ the increase of the contact angle is no longer pinned, and when the contact angle $\pi-\theta_{c}$ has been reached, the droplet can grow further with this contact angle and increasing thereby its contact line $b_{d r o p}(\tau)$ beyond $b$ with increasing time. Thus, the two critical fields $H_{b}^{\text {crit }}$ and $H_{b}^{\text {spin }}$ simply correspond to the cases $R^{*}=b /\left(2 \sin \left(\theta_{c}\right)\right)$ and $R^{*}=b / 2$, respectively. Note that in panel (c) the length of the inhomogeneity in the upper sketch was taken a factor two larger than in the medium and lower sketches for the sake of clarity. Panel (d) shows plots of the excess free energy relative to the wall without droplet $\Delta F(R) v s R$ [see Eq. (A12), as obtained for different fields corresponding to the regimes shown in panels (a)-(c)]. Theoretical curves are obtained by taken $\theta_{c}=35^{\circ}$ and $f_{\text {int }}=1.7987$ in order to illustrate the expected behavior for $t=0.40$. The chosen fields are then suitable to describe the case of an inhomogeneity of extension $b=21$ such that $b /\left(2 \sin \left(\theta_{c}\right)\right)=18.31$; see the vertical dashed line. The values of $R^{*}$ corresponding to the selected fields are shown along the horizontal axis. More details in the text. 
length $b$, with contact angles exceeding the equilibrium value, $\theta_{c}<\theta<\pi / 2$ (cf. Fig. 20). Assuming that these droplets still have circle cut shape, we have predicted that these droplets become unstable for $H_{b}^{\text {spin }}=f_{\text {int }} /\left(b m_{\text {coex }}\right)$, and we have obtained an approximation for $\tilde{M}\left(t, b H_{b}\right)$ in this regime [Fig. 9(a)]. Note that further growth of the droplets with baseline $b_{\text {drop }}>b$ requires that the contact angle grows up to $\pi-\theta_{c}$, and in the regime $H_{b}^{\text {crit }}<H_{b}<H_{b}^{\text {spin }}$, this is hindered by a free energy barrier $\Delta F_{2}$; see Fig. 13 and Eq. (26). Since this barrier is only of the order of a few $k_{B} T$ when $\theta$ has reached about $70^{\circ}$, pinned droplets with $70^{\circ}<\theta<\pi / 2$ actually were not observed; droplets with a shape as sketched in the lower part of Fig. 20 then appear in a second nucleation event and grow to complete the phase transformation. So the actual limit of metastability $H_{b}^{\max }$ of pinned droplets is somewhat smaller than $H_{b}^{\text {spin }}$, e.g., (for $b=51$ ) $H_{b}^{\max } b \simeq 1.71$, while $H_{b}^{\text {spin }} b \simeq 1.7987$.

Thus, an unexpectedly rich behavior concerning nucleation at a chemically inhomogeneous substrate has been found within the context of the grand canonical ensemble used in our calculations (i.e., when the pressure of the fluid or equivalently the magnetic field of the Ising model is given as a control parameter). We have shown that this scenario differs from that corresponding to the canonical ensemble (i.e., when the volume in $d=3$ or the area in $d=2$ of the droplets is taken as a control parameter). In order to acquaint the reader with a clear description of the relevant findings reported in this paper, we have summarized and discussed our results in Fig. 18. In this way we addressed the relevant regimes encountered in our study performed in the framework of the grand canonical ensemble: (a) The regime $H_{b}<H_{b}^{\text {crit }}$ where no nucleation is possible. (b) The regime $H_{b}^{c r i t}<H_{b}<H_{b}^{\text {spin }}$, where the droplet grows with contact angle $\theta_{c}$ until its baseline matches the length of the inhomogeneity and then subsequently grows by keeping its baseline constant but increasing the contact angle. Finally, the regime (c) corresponds to larger fields $H_{b}>H_{b}^{\text {spin }}$ that lie beyond the stability limit so that the droplets can grow with the baseline larger than the length of the inhomogeneity and contact angle $\pi-\theta_{c}$. Furthermore, all these three regimes are properly identified with the corresponding free energy functions $F(R)$ shown in panel (d) of Fig. 18.

\section{ACKNOWLEDGMENTS}

E.V.A. is grateful to the Alexander von Humboldt foundation and to the Deutsche Forschungsgemeinschaft (DFG, SFB TRR 146) for partial support of his research stays at the Institut für Physik of the Johannes Gutenberg Universität Mainz. Also, M.L.T. and E.V.A. are grateful to the CONICET and UNLP (Argentina) for financial support.

\section{APPENDIX A: HETEROGENEOUS NUCLEATION ON HOMOGENEOUS AND CHEMICALLY INHOMOGENEOUS SUBSTRATES: PHENOMENOLOGICAL THEORY}

We consider here a fluid in $d=2$ dimensions exposed to a (one-dimensional) flat wall, under conditions of partial wetting. For simplicity, we shall consider explicitly only the nearest-neighbor lattice gas (Ising) model and the wall is then oriented along the $x$-direction of the lattice; the Ising spins adjacent to this wall then have a row of missing neighbors but experience a surface field $H_{w 3}$ chosen such that the liquid phase of the lattice gas model is favored; unlike Fig. 1, we consider first the case where this boundary field is homogeneous independent of the coordinate $x$. In the semi-infinite system at zero bulk field $H_{b}=0$, the vapor phase can be stable (due to a suitable boundary field at $y=L \rightarrow \infty$ ), and near the boundary where $H_{w 3}$ acts only a liquid film of the average thickness of order $\xi_{\perp}$ is stabilized; $\xi_{\perp}$ is the perpendicular correlation length of the wetting transition, and in the regime of partial wetting, this is a microscopic length, i.e., of the order of the lattice spacing in the lattice gas model.

Note that in the grand canonical ensemble (temperature $T$ and bulk field $H_{b}$ chosen as control variables) liquid droplets in full thermal equilibrium can exist neither in the bulk nor attached to the wall, even if a nonzero bulk field favoring the liquid phase is switched on. However, metastable droplets can exist only for a finite "lifetime" and we shall address this case using concepts of the theory of heterogeneous nucleation. Thus, it is useful to recall that stable liquid droplets of macroscopic size do exist when we consider the canonical ensemble, choosing the density of the vapor $\rho$ in between the density of coexisting vapor $\left(\rho_{v}\right)$ and liquid $\left(\rho_{l}\right)$ phases. The area fraction of the liquid is then given by the lever rule, $X=\left(\rho-\rho_{v}\right) /\left(\rho_{l}\right.$ $\left.-\rho_{v}\right)$, and the shape of the liquid domain does depend on the choice of boundary conditions. In our case, for small $X$ we obtain a wall attached droplet having the shape of a circle cut with baseline $b_{d r o p}$ given in terms of the droplet radius $R$ and contact angle $\theta_{c}$ by

$$
b_{\text {drop }}=2 R \sin \left(\theta_{c}\right)
$$

and the contact angle for an isotropic interface tension $f_{\text {int }}$ between the coexisting vapor and liquid phases is given by Young's equation, namely,

$$
f_{\text {int }} \cos \left(\theta_{c}\right)=f_{w v}-f_{w l}
$$

where $f_{w v}, f_{w l}$ are the surface excess free energy densities of the vapor phase $\left(f_{w v}\right)$ and liquid phase $\left(f_{w l}\right)$ due to the wall. Actually, Eq. (A2) is valid for a fluid in continuous space, but not for the lattice gas model, where the interface tension $f_{\text {int }}(\theta)$ depends on the angle $\theta$ between the interface normal and the $x$ axis of the lattice. Then Eq. (A2) needs to be replaced by

$$
f_{\text {int }}\left(\theta_{c}\right) \cos \left(\theta_{c}\right)-\left.\sin \left(\theta_{c}\right) \frac{d f_{i n t}\left(\theta_{c}\right)}{d \theta_{c}}\right|_{\theta=\theta_{c}}=f_{w v}-f_{w l} .
$$

While in the isotropic case straightforward geometric considerations yield the area $(A)$ of the circle cut as

$A=R^{2}\left(\theta_{c}-\frac{1}{2} \sin \left(2 \theta_{c}\right)\right)=\frac{1}{4} b_{d r o p}^{2}\left(\frac{\theta_{c}}{\sin ^{2}\left(\theta_{c}\right)}-\frac{\cos \left(\theta_{c}\right)}{\sin \left(\theta_{c}\right)}\right)$,

and the length of the vapor-liquid interface line is

$$
l_{l v}=2 R \theta_{c}=b_{d r o p} \frac{\theta_{c}}{\sin \left(\theta_{c}\right)},
$$

finding the droplet shape for the anisotropic case is less straightforward.

In the bulk, this problem is solved in terms of the well known Wulff construction, which for the $d=2$ Ising model 
can be worked out explicitly, and the shape of the droplet is given by the equation ${ }^{61,62}$

$$
\cosh (\tilde{x})+\cosh (\tilde{y})=\cosh \left(2 J / k_{B} T\right) / \tanh \left(2 J / k_{B} T\right),
$$

where $\tilde{x}$ and $\tilde{y}$ are the $x, y$ coordinates of the curve describing the droplet shape. Equation (A6) interpolates smoothly between a square shape (for $T \rightarrow 0$ ) and a circle (for $T \rightarrow T_{c b}$ ). When we inscribe a circle that touches the actual shape at $\tilde{x}=0$ and at $\tilde{y}=0$, it has a radius $R_{\text {in }}$ given by

$$
R_{i n}=\operatorname{arccosh}\left[\cosh \left(2 J / k_{B} T\right) / \tanh \left(2 J / k_{B} T\right)-1\right] .
$$

As an example, we hence plotted Eq. (A6), in Fig. 19, together with the inscribed circle of radius $R_{i n}$ evaluated at $t=T / T_{c b}$ $=0.40$ since for this choice of the reduced temperature most of our simulations were made. We found that the deviations from the spherical shape are already rather minor, and this justifies our neglect of these anisotropy effects, at least as a first approximation. The solution of Eq. (A6) reduces to the equation of a circle near $T_{c b}$, where $\tilde{x} \rightarrow 0$ and $\tilde{y} \rightarrow 0$ and hence

$$
\tilde{x}^{2}+\tilde{y}^{2}=2 \cosh \left(2 J / k_{B} T\right) / \tanh \left(2 J / k_{B} T\right)-4,
$$

recalling that $\cosh \left(2 J / k_{B} T_{c b}\right)=2^{\frac{1}{2}}, \sinh \left(2 J / k_{B} T_{c b}\right)=1$, and hence $R_{i n} \rightarrow 0$ as well. The solution for the wall-attached droplet is then given by the Winterbotton construction; ${ }^{63}$ i.e., we have to cut the droplet shown in Fig. 19 by a horizontal straight line such that the angle of the tangent is $\theta_{c}$ as given by Eq. (A3). The linear dimensions $\tilde{y}_{c u t}$ and $R_{\text {in }}$ then follow from the condition that the area above the cut yields the desired area fraction $X$.

However, for the sake of simplicity, we shall ignore these anisotropy effects in the following, working with droplets of circular shape only. But even then there is one fundamental problem: there is no physical reason for the $x$-coordinate of the center of mass of the droplet to coincide with the origin of the coordinate system. In fact, this center of mass coordinate can be anywhere on the $x$-axis when the boundary field is homogeneous, independent of $x$. Even in the inhomogeneous case, the droplets are only on average centered in the middle of the inhomogeneity, as, e.g., can be qualitatively observed

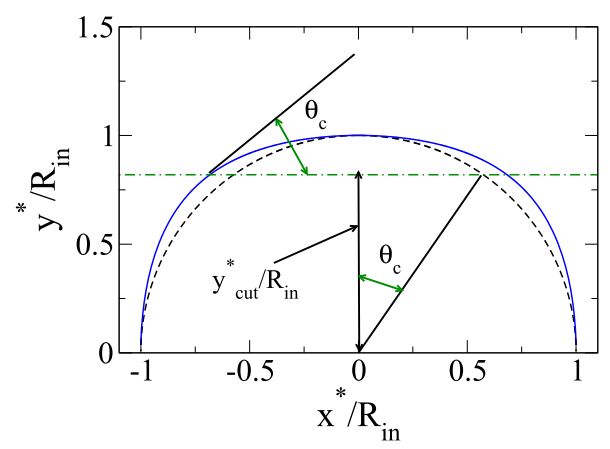

FIG. 19. The full line shows the shape of the droplet given by the Eq. (A6), while the dashed line shows the inscribed circle of radius $R_{\text {in }}$ given by Eq. (A7) and evaluated at $t=T / T_{c b}=0.40$, i.e., $R_{i n}=1.98094$. The horizontal dasheddotted line shows the location of the circle cut line placed at a distance $y_{\text {cut }}^{*} / R_{\text {in }}=\cos \left(\theta_{c}\right)$ from the origin. The contact angle of the droplet at the intersection between the circle and the cut line, which is the angle that the droplet makes with the substrate, is also shown (notice that $\theta_{c}=35^{\circ}$ has been the choice in this example). in the snapshots of Fig. 2. This fact creates a translational entropy contribution $k_{B} T \ln (M)$ for the droplet, where $1 \leq x \leq$ $M$ in our finite lattice of length $M$ in the homogeneous case. Similar translational entropy contributions are known to hamper the numerical study of interfacial free energies. ${ }^{71,72}$ Thus, in a straightforward simulation study of the present problem, the droplet would diffuse along the $x$-axis and its density profile $\rho(x, y)$ would be completely smeared out, until only the average translationally invariant density profile $\rho_{a v}(y)$ is left, containing little information on the droplet. Thus a "demon" would be needed to constrain the sampling of configurations such that in each microstate of the system that is sampled the droplet center of mass has its $x$-coordinate in the origin. Practical implementation of such a constraint is not completely trivial since the size and the shape of the droplet due to their nanoscale dimensions is strongly fluctuating (cf. Fig. 2).

We now consider the main subject of interest of the present paper, namely, a boundary condition of the type shown in Fig. 1, where the surface field $H_{w 3}$ acts only over a distance $b$ along the $x$-axis, while in the remaining boundary a field $H_{w 2}$ $=-\left|H_{w 3}\right|$ acts, and hence the contact angle $\theta_{c}^{\prime}=\pi-\theta_{c}$ applies.

In $d=3$ dimensions in the canonical ensemble, this situation has already been considered by Lipowsky et al. ${ }^{65-67}$ They pointed out that three regimes need to be distinguished, namely: (I) $b_{d r o p}<b$, (II) $b_{d r o p}=b$, and (III) $b_{\text {drop }}>b$; see Fig. 20 adapted to our $d=2$ dimensional case. Here the area $A$ of the wall attached droplet is the control parameter that is varied: Sufficiently small area taken by the liquid baseline $b_{\text {drop }}$ that will result from $A$ and $\theta_{c}$ via Eq. (A4) will be in the regime I, and the $x$-coordinate of the center of mass of the droplet can be anywhere in the interval from $x=-(b-$ $\left.b_{\text {drop }}\right) / 2$ to $x=+\left(b-b_{\text {drop }}\right) / 2$. Unlike Lipowsky et al.,${ }^{65-67}$ we do not assume that the droplet is exactly centered at $x=0$, the center of the inhomogeneity of the wall, which is our origin. This center certainly is the most probable position, but there will be a broad probability distribution for this center of mass coordinate, and when we consider the average density profile $\rho(x, y)$ obtained by convoluting the density profile of the droplet with baseline $b_{d r o p}$ and contact angle $\theta_{c}$ with this probability distribution, a density distribution $\rho_{\text {ave }}(x, y)$ must result that is considerably flattened in comparison with $\rho(x, y)$. From $\rho_{\text {ave }}(x, y)$, one would obtain an effective contact angle $\theta_{c}^{\text {eff }}$ that clearly will be much smaller than the correct one, if $b_{d r o p} \ll b$. This entropic effect was disregarded by Lipowsky et al. ${ }^{65-67}$ but clearly must be present in our simulations and thus hampers their interpretation. It is tempting to associate the small values of $\theta_{e f f}$ in Figs. 6 and 7 observed for $l_{e f f}<b$ with this flattened profiles due to the fluctuations in the center of mass position of small wall-attached droplets.

The most interesting situations of course are found when $b_{d r o p}$ as given by Eqs. (A1) and (A4) has reached the value $b_{d r o p}=b$ : then the prediction is that further increase of $A$ does not cause a further growth of $b_{\text {drop }}$. Rather, what happens is a growth of the contact angle $\theta$ of the droplet from the value $\theta_{c}$ given by Young's equation to a larger value, satisfying an equation analogous to Eq. (A4), namely,

$$
A=\frac{1}{4} b^{2}\left(\frac{\theta}{\sin ^{2}(\theta)}-\frac{\cos (\theta)}{\sin (\theta)}\right), \quad \theta_{c}<\theta<\theta_{c}^{\prime} .
$$



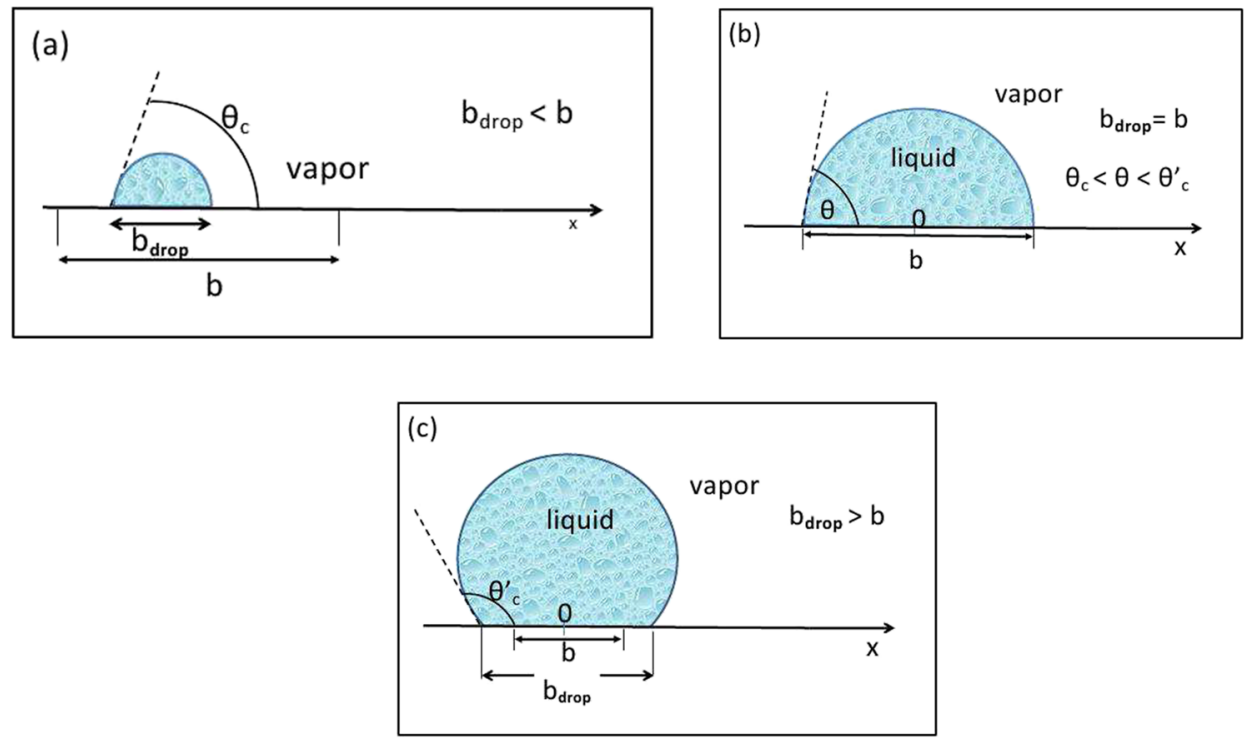

FIG. 20. Sketches of droplets corresponding to the three relevant regimes considered by Lipowsky et al., ${ }^{65-67}$ conveniently adapted to our twodimensional case, namely: (I) $b_{\text {drop }}<$ $b$ (a); (II) $b_{d r o p}=b$ (b); and (III) $b_{d r o p}$ $>b(\mathrm{c})$, where $b_{d r o p}$ is the baseline of the droplet in contact with the substrate, $b$ is the length of the heterogeneity, and $\theta_{c}$ is the contact angle. More details in the text.
Thus in a sense the interface between the liquid and vapor is pinned at the points $x= \pm b / 2$ when $A$ has increased up to the value where Eq. (A9) yields $\theta=\theta_{c}^{\prime}\left(=\pi-\theta_{c}\right.$, in our case), depinning of the interface from the inhomogeneities of the boundary occurs, and $\theta$ stays at $\theta_{c}^{\prime}$, while $b_{\text {drop }}>b$. Again Lipowsky et al. ${ }^{65-67}$ have assumed that the $x$-coordinate of the center of mass of the droplet is at $x=0$, but we maintain that again fluctuations will occur. However, the region $b_{d r o p}$ in between the two contact points of the interface will always encompass the region of the inhomogeneity, from $x=-b / 2$ to $x=+b / 2$, and the average position of the center of mass of the droplet will hence have the $x$-coordinate $x=0$.

When we now turn to the description in the grand canonical ensemble, we note that a correspondence to the droplet configurations discussed for the canonical ensemble can exist only when the droplet configurations in the grand canonical ensemble are still metastable.

For the problem without boundary inhomogeneity, we have the standard problem of heterogeneous nucleation at the wall. The free energy cost of the forming droplet is written as the excess free energy relative to the wall without droplet, namely,

$$
\Delta F_{\text {drop }}=-2 m_{\text {coex }} H_{b} A+\Delta F_{\text {int }},
$$

where $m_{\text {coex }}$ is the spontaneous magnetization of the Ising model, and $A$ is given by Eq. (A4) and $\Delta F_{\text {int }}$ becomes

$$
\begin{aligned}
\Delta F_{\text {int }} & =2 R f_{\text {int }} \theta_{c}+\left(f_{w l}-f_{w v}\right) 2 R \sin \left(\theta_{c}\right) \\
& =2 f_{\text {int }} R\left[\theta_{c}-\frac{1}{2} \sin \left(2 \theta_{c}\right)\right],
\end{aligned}
$$

where Eq. (A2) was used. Equations (A10) and (A11) yield

$$
\Delta F_{\text {drop }}=\left[\theta_{c}-\frac{1}{2} \sin \left(2 \theta_{c}\right)\right]\left[-2 m_{\text {coex }} H_{b} R^{2}+2 f_{\text {int }} R\right] .
$$

Minimizing $\Delta F_{\text {drop }}$ with respect to $R$ yields

$$
R^{*}=f_{\text {int }} /\left(2 m_{\text {coex }} H_{b}\right), \quad \Delta F_{\text {het }}^{*}=\Delta F_{\text {homo }}^{*} f_{V T}\left(\theta_{c}\right),
$$

where $\Delta F_{\text {homo }}^{*}$ is the standard result for the free energy barrier against homogeneous nucleation in $d=2$ dimensions

$$
\Delta F_{\text {homo }}^{*}=\frac{\pi}{2} f_{\text {int }}^{2} /\left(m_{\text {coex }} H_{b}\right)
$$

and $f_{V T}\left(\theta_{c}\right)$ is the analog of the well-known Volmer-Turnbull function in $d=2$ dimensions, given by

$$
f_{V T}\left(\theta_{c}\right)=\frac{1}{\pi}\left[\theta_{c}-\frac{1}{2} \sin \left(2 \theta_{c}\right)\right] .
$$

Note that $f_{V T}\left(\theta_{c}\right) \approx \frac{2}{3 \pi} \theta_{c}^{3}$ for $\theta_{c} \rightarrow 0$, when complete wetting begins. It turns out, of course, that use of $R^{*}$ $=f_{\text {int }} /\left(2 m_{\text {coex }} H_{b}\right)$ in Eq. (A1) yields $b_{\text {drop }} \ll b$ only for rather large fields. All the data where the metastable droplets are encountered do not fall in this regime, as expected.

\section{APPENDIX B: PINNED DROPLETS: COMPARING THE TWO- AND THE THREE-DIMENSIONAL CASES}

While the numerical simulation work exclusively has addressed the case of a two-dimensional system with a onedimensional boundary where the positive surface field (favoring the liquid phase of the lattice gas) acts on a length $b$, it is also instructive to consider the three-dimensional case, where the positive surface field acts on a circular heterogeneity with radius $r$. For the sake of clarity, the geometry of the pinned droplet is sketched in Fig. 21.

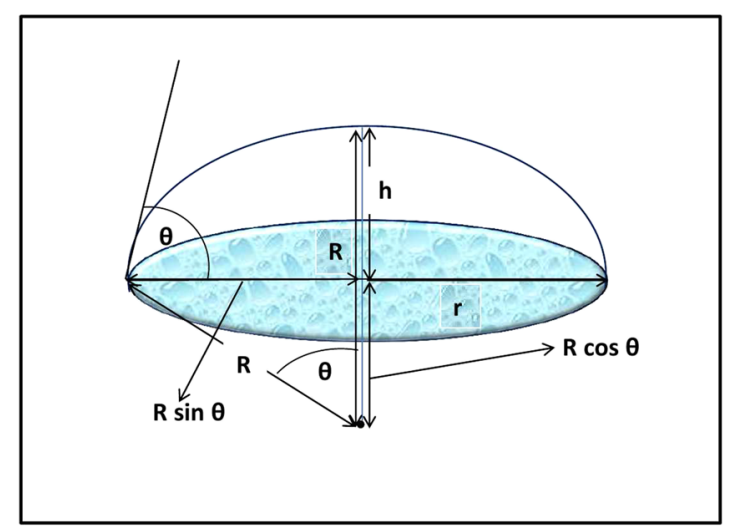

FIG. 21. Geometrical description used to construct a spherical cap droplet, where $\theta$ is the angle that the droplet makes with the substrate. More details in the text. 

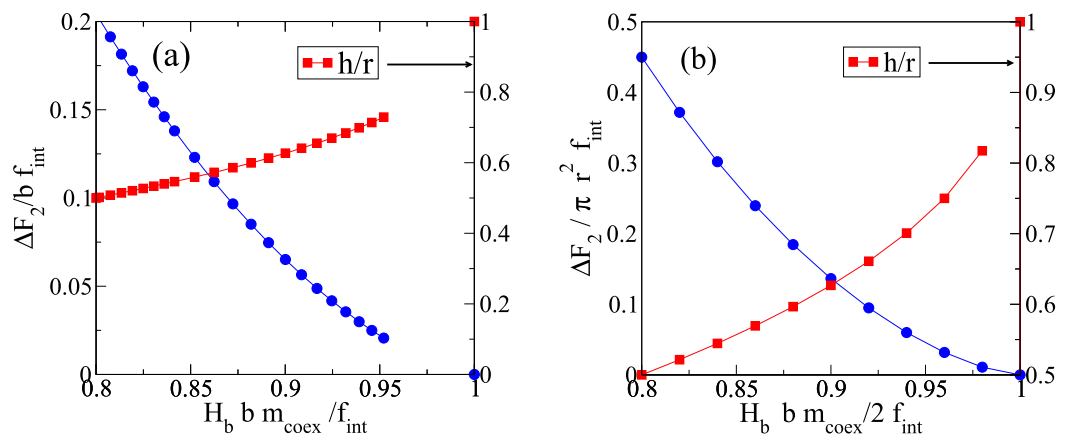

FIG. 22. Comparison of the free energy barrier of the depinning transition of pinned droplets for the two- and the three-dimensional cases shown in panels (a) and (b), respectively. For $d=3$, we assume $b=2 r$; see Fig. 21. The left ordinate scales refer to the scaled free energy barrier and the right scales refer to $h / r$, respectively. More details in the text.

The radius of curvature of the sphere-cap shaped droplet is $R$. Then

$$
r=R \sin (\theta), \quad h=R(1-\cos (\theta)),
$$

where it is convenient to express all quantities in terms of the height $h$ of the droplet above the substrate. The angle $\theta$ that the droplet makes with the substrate can be in the range

$$
\theta_{c} \leq \theta \leq \pi-\theta_{c},
$$

where $\theta_{c}$ is the contact angle given by Young's equation. Notice that only for angles in the quoted range droplets with basal radius $r$ exist; however, only for $\theta \leq \pi / 2$ such droplets are metastable, while for $\pi / 2 \leq \theta \leq \pi-\theta_{c}$ they are unstable.

Now the volume of the sphere cap is

$$
V=\frac{\pi h}{6}\left(3 r^{2}+h^{2}\right),
$$

and also the basis surface is $\pi r^{2}$, while the upper surface is

$$
A_{u}=\pi\left(r^{2}+h^{2}\right) \text {. }
$$

So, the free energy difference of the droplet of height $h$ relative to a disk-shaped droplet of radius $r$ and height $h=0$ is (the choice of this reference state is arbitrary, of course)

$$
\Delta F=f_{\text {int }} A_{u}-2 m_{\text {coex }} H_{b} V,
$$

where $H_{b}$ is the bulk field. Then, by using Eqs. (B3) and (B4), one obtains

$$
\Delta F=f_{\text {int }} \pi\left(r^{2}+h^{2}\right)-m_{\text {coex }} H_{b} \frac{\pi}{3} h\left(3 r^{2}+h^{2}\right) .
$$

It is convenient to find the extrema of $\Delta F$ simply as a function of $h$; then

$$
(\partial(\Delta F) / \partial h)_{H_{b}}=0
$$

yields

$$
h^{2}-2 h \frac{f_{\text {int }}}{m_{\text {coex }} H_{b}}+r^{2}=0
$$

such that in terms of $\tilde{H}_{b}=H_{b} r m_{\text {coex }} / f_{\text {int }}$ one finds for $\tilde{H}_{b}<1$ two solutions, namely,

$$
\frac{h}{r}=\tilde{H}_{b}^{-1} \pm \sqrt{\left(\tilde{H}_{b}^{-2}-1\right)} .
$$

The minus sign yields the free energy minimum, corresponding to the pinned droplet, while the plus sign corresponds to a surface free energy maximum, and the corresponding angle $\theta$ can be read off from

$$
\tan \left(\frac{\theta}{2}\right)=\frac{1-\cos (\theta)}{\sin (\theta)}=\frac{h}{r} .
$$

The limiting case $\tilde{H}_{b}=1$ means $h / r=1, \theta=\pi / 2$, i.e., a semispherical droplet. The free energy function can be written as

$$
\Delta F / f_{i n t} \pi r^{2}=\frac{2}{3}\left(\tilde{H}_{b}^{-2} \pm \tilde{H}_{b}^{-1} \sqrt{\left(\tilde{H}_{b}^{-2}-1\right)} \mp \tilde{H}_{b} \sqrt{\left(\tilde{H}_{b}^{-2}-1\right)}\right)
$$

and hence the barrier for the depinning transition of the pinned droplet becomes

$$
\Delta F_{2} / f_{i n t} \pi r^{2}=\frac{4}{3} \tilde{H}_{b}\left(\tilde{H}_{b}^{-2}-1\right)^{3 / 2} .
$$

From this calculation, it is obvious that the mathematics in $d=3$ is even simpler than in $d=2$ since the use of $h$ instead of the angle $\theta$ makes the description of $\Delta F$ [cf. Eq. (B5)] very simple. In $d=2$, Eq. (B10) also holds, but Eq. (20) shows that both $\theta$ and $\sin (2 \theta)$ enter in the free energy expression, so no simple formula for $\Delta F(h)$ in $d=2$ can be written down. When one works out $h / r$ and $\Delta F_{2}$ in both $d=2$ and $d=3$, one notes a very similar behavior: near the point $\tilde{H}_{b}=1$, the barrier vanishes like $\left(1-\tilde{H}_{b}^{-1}\right)^{3 / 2}$, i.e., with a vanishing slope, and $h / r$ reaches the semicircle or semisphere configuration with a square-root cusp. Figure 22 shows a comparison of the free energy barrier of the depinning transition of pinned droplets for the two- and the three-dimensional cases.

${ }^{1}$ L. D. Landau and E. M. Lifshitz, Statistical Physics (Pergamon Press, Oxford, 1958)

${ }^{2}$ K. Binder, Rep. Prog. Phys. 50, 783 (1987).

${ }^{3}$ Nucleation, edited by A. C. Zettlemoyer (M. Dekker, New York, 1969).

${ }^{4}$ P. G. Debenedetti, Metastable Liquids (Princenton University Press, Princenton, 1996).

${ }^{5}$ D. Kashchiev, Nucleation: Basic Theory with Applications (ButterworthHeinemann, Oxford, 2000).

${ }^{6}$ Nucleation, Comptes Rendus Physique, edited by S. Balibar and J. Villain (Academie des Sciences, Paris, 2006), Vol. 7, special issue 9-10.

${ }^{7}$ D. Beysens, C. R. Phys. 7, 1082 (2006); D. Beysens, V. Pruvost, and B. Pruvost, J. Arid Environ. 135, 90 (2016).

${ }^{8} \mathrm{H}$. Biloni, in Physical Metallurgy, edited by R. W. Cahen and P. Haasen (North-Holland, Amsterdam, 1983), p. 477.

${ }^{9}$ M. Volmer, Kinetik der Phasenbildung (Th. Steinkopff, Dresden, Leipzig, 1939).

${ }^{10}$ D. Turnbull, J. Appl. Phys. 21, 1022 (1950).

${ }^{11}$ D. Turnbull, J. Chem. Phys. 18, 198 (1950).

${ }^{12}$ D. Turnbull and B. Vonnegut, Ind. Eng. Chem. 44, 1292 (1952).

${ }^{13}$ N. H. Fletcher, J. Chem. Phys. 29, 572 (1958).

${ }^{14}$ N. H. Fletcher, J. Chem. Phys. 31, 1136 (1959)

${ }^{15}$ N. H. Fletcher, J. Chem. Phys. 38, 237 (1963).

${ }^{16}$ V. Talanquer and D. W. Oxtoby, J. Chem. Phys. 104, 1483 (1996).

${ }^{17}$ V. Talanquer and D. W. Oxtoby, J. Chem. Phys. 114, 2793 (2001).

${ }^{18}$ T. V. Bykov and X. C. Zheng, J. Chem. Phys. 117, 1851 (2002).

${ }^{19}$ R. P. Sear, J. Phys.: Condens. Matter 14, 3693 (2002).

${ }^{20}$ S. Auer and D. Frenkel, Phys. Rev. Lett. 91, 015703 (2003). 
${ }^{21}$ A. Cacciuto, S. Auer, and D. Frenkel, Nature 428, 404 (2004).

${ }^{22}$ A. Cacciuto and D. Frenkel, Phys. Rev. E 72, 041604 (2005).

${ }^{23}$ E. A. Ustinov, and D. D. Do, J. Phys. Chem. 109, 11653 (2005).

${ }^{24}$ R. P. Sear, J. Phys. Chem. B 110, 4985 (2006).

${ }^{25}$ T. V. Bykov and X. C. Zheng, J. Chem. Phys. 125, 144515 (2006).

${ }^{26}$ A. J. Page and R. P. Sear, Phys. Rev. Lett. 97, 065701 (2006).

${ }^{27}$ R. P. Sear, J. Phys.: Condens. Matter 19, 033101 (2007).

${ }^{28}$ D. Winter, P. Virnau, and K. Binder, J. Phys.: Condens. Matter 21, 464118 (2009).

${ }^{29}$ D. Winter, P. Virnau, and K. Binder, Phys. Rev. Lett. 103, 225703 (2009).

${ }^{30}$ D. Deb, A. Winkler, P. Virnau, and K. Binder, J. Chem. Phys. 136, 134710 (2012).

${ }^{31}$ F. Schmitz, P. Virnau, and K. Binder, Phys. Rev. E 87, 053302 (2013).

${ }^{32}$ K. S. Singha, P. K. Das, and B. Maiti, J. Chem. Phys. 142, 104706 (2015).

${ }^{33}$ K. Binder and P. Virnau, J. Chem. Phys. 145, 211701 (2016).

${ }^{34}$ Handbook of Nanophysics: Principles and Methods, edited by K. D. Sattler (CRC Press, Boca Raton, 2011); Wiley, New York, 1995.

${ }^{35}$ Nanoscale Liquid Interfaces: Wetting, Patterning, and Force Microscopy at the Molecular Scale, edited by Th. Ondarçuhu and J.-P. Aimé (Pan Stanford Publishing Pte. Ltd., Stanford, 2013).

${ }^{36}$ K. Binder and D. Stauffer, Adv. Phys. 25, 343 (1976).

${ }^{37}$ P. A. Rikvold, H. Tomita, S. Miyashita, and S. W. Sides, Phys. Rev. E 49, 5080 (1994)

${ }^{38}$ B. A. Berg and S. Dubey, Phys. Rev. Lett. 100, 165702 (2008).

${ }^{39}$ E. N. M. Cirillo and J. L. Lebowitz, J. Stat. Phys. 90, 211 (1998).

${ }^{40}$ M. Rauscher and S. Dietrich, Annu. Rev. Mater. Sci. 38, 143 (2008).

${ }^{41} \mathrm{M}$. Rauscher and S. Dietrich, Handbook of Nanophysics: Principles and Methods (CRC Press, Boca Raton, 2017), edited by K. D. Sattler, Chap. 11.

${ }^{42}$ S. Dietrich, M. Rauscher, and M. Napiorkowski, Nanoscale Liquid Interfaces: Wetting, Patterning, and Force Microscopy at the Molecular Scale (Pan Stanford Publishing Pte. Ltd., Stanford, 2013), Chap. 3.

${ }^{43} \mathrm{~K}$. Binder and D. W. Heermann, Monte Carlo Simulation in Statistical Physics. An Introduction, 5th ed. (Springer, Berlin, 2010).

${ }^{44}$ D. Bonn, J. Eggers, J. Indekeu, J. Meunier, and E. Rolley, Rev. Mod. Phys. 81, 739 (2009).

${ }^{45}$ K. Binder, D. P. Landau, and S. Wansleben, Phys. Rev. B 40, 6971 (1989).
${ }^{46}$ E. V. Albano and K. Binder, Phys. Rev. Lett. 109, 036101 (2012).

${ }^{47}$ P. Bryk and K. Binder, Phys. Rev. E 88, 030401 (2013).

${ }^{48}$ L. Onsager, Phys. Rev. 65, 117 (1944).

${ }^{49}$ C. N. Yang, Phys. Rev. 85, 808 (1952).

${ }^{50}$ D. B. Abraham, Phys. Rev. Lett. 44, 1165 (1980).

${ }^{51}$ D. B. Abraham, in Phase Transitions and Critical Phenomena, edited by C. Domb and J. L. Lebowitz (Academic Press, London, 1986), Vol. 10, Chap. 1 .

${ }^{52}$ D. B. Abraham, J. De Coninck, and F. Dunlop, Phys. Rev. B 39, 4708 (1989).

${ }^{53}$ M. E. Fisher, J. Stat. Phys. 34, 667 (1984).

${ }^{54}$ X.-T. Wu, D. B. Abraham, and J. O. Indekeu, Phys. Rev. Lett. 116, 046101 (2016).

${ }^{55}$ M. L. Trobo, E. V. Albano, and K. Binder, Phys. Rev. E 93, 052805 (2016).

${ }^{56}$ K. Brendel, G. T. Barkema, and H. van Beijeren, Phys. Rev. E 71, 031601 (2005).

${ }^{57}$ H. Tomita and S. Miyashita, Phys. Rev. B 46, 8886 (1992).

${ }^{58}$ P. Jakubczyk, M. Napiórkowski, and A. O. Parry, Phys. Rev. E 74, 031608 (2006).

${ }^{59}$ P. Jakubczyk and M. Napiórkowski, J. Phys. A: Math. Theor. 40, 2363 (2007).

${ }^{60}$ M. P. A. Fisher, D. S. Fisher, and J. D. Weeks, Phys. Rev. Lett. 48, 368 (1982).

${ }^{61}$ R. P. K. Zia and J. E. Avron, Phys. Rev. B 25, 2042 (1982).

${ }^{62}$ C. Rottman and M. Wortis, Phys. Rep. 103, 59 (1984).

${ }^{63}$ W. L. Winterbottom, Acta Metall. 15, 303 (1967).

${ }^{64}$ L. Schimmele, M. Napiórkowski, and S. Dietrich, J. Chem. Phys. 127, 164715 (2007).

${ }^{65}$ P. Lenz and R. Lipowsky, Phys. Rev. Lett. 80, 1920 (1998).

${ }^{66}$ R. Lipowsky, P. Lenz, and P. S. Swain, Colloids Surf., A 161, 3 (2000).

${ }^{67}$ R. Lipowsky, M. Brinkmann, R. Dimova, T. Franke, J. Kierfeld, and X. Zhang, J. Phys.: Condens. Matter 17, S537 (2005).

${ }^{68}$ N. J. Günther, D. A. Nicole, and D. J. Wallance, J. Phys. A: Math. Gen. 13, 1755 (1980).

${ }^{69}$ S. Ryu and W. Cai, Phys. Rev. E 81, 030601(R) (2010).

${ }^{70}$ A. Tröster, F. Schmitz, P. Virnau, and K. Binder. J. Phys. Chem. B (in press).

${ }^{71}$ F. Schmitz, P. Virnau, and K. Binder, Phys. Rev. Lett. 112, 126701 (2014).

${ }^{72}$ F. Schmitz, P. Virnau, and K. Binder, Phys. Rev. E 90, 012128 (2014). 